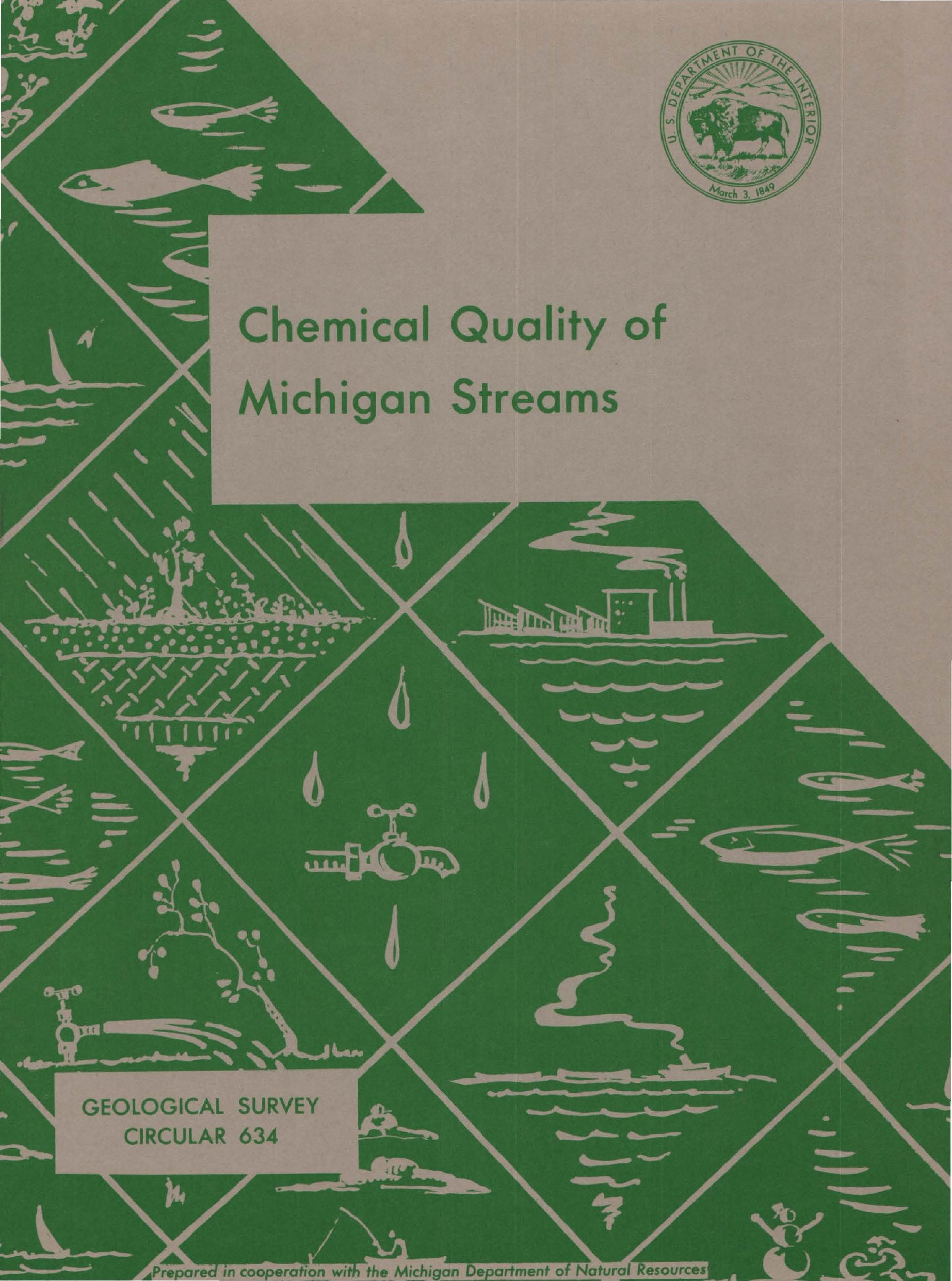





\section{Chemical Quality of Michigan Streams}

By Warren W. Wood

GEOLOGICAL SURVEY CIRCULAR 634

Prepared in cooperation with the Michigan Department of Natural Resources

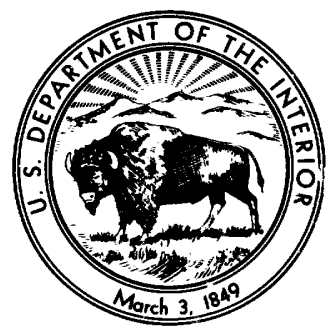




\section{United States Department of the Interior}

WALTER J. HICKEL, Secretary

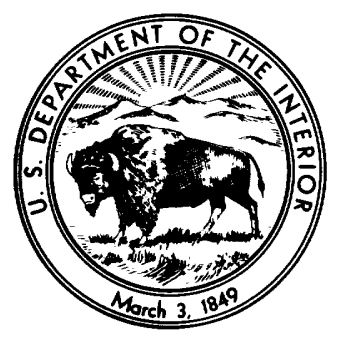

\section{Geological Survey}

William T. Pecora, Director

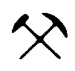




\section{CONTENTS}

\begin{tabular}{|c|c|c|}
\hline & \\
\hline & & \\
\hline & \\
\hline & \multicolumn{2}{|c|}{$\begin{array}{l}\text { Page } \\
\text { Definition of terms } \\
\text { Abstract } \\
\text { Introduction }\end{array}$} \\
\hline \multicolumn{3}{|r|}{ lity of streams ... } \\
\hline \multicolumn{3}{|c|}{\begin{tabular}{lll}
$\ldots$. & 1 & Conclusions \\
\hdashline.. & 3 & Selected references
\end{tabular}} \\
\hline
\end{tabular}

\section{ILLUSTRATIONS}

Figure 1. Map showing locations and identification numbers of sampling sites

Page

2

2-9. Maps showing-

2,3. Concentration of dissolved solids in streams under lowand high-flow conditions

6,7. Concentration of chloride in streams under low- and high-flow conditions

8,9. Concentration of sulfate in streams under low- and high-flow conditions

10-12. Map showing-

10. Average annual runoff, in inches, 1951-60

12

11. Average annual lake evaporation, in inches, 1946-55 ......

12. Mean annual precipitation, in inches, 1931-55

\section{TABLE}

TABLE 1. Chemical analyses of samples collected from Michigan streams under low- and high-flow conditions, 1967 


\section{DEFINITION OF TERMS}

Dissolved solids. Consist mainly of the dissolved mineral constitutents in water and are represented by the residue that remains after filtration, evaporation, and $d * y-$ ing at a temperature of $180^{\circ} \mathrm{C}$.

Hardness. A property of water which causes an increase in the amount of soap that is needed to produce foam or lather. Hardness is produced almost completely by the presence of calcium and magnesium salts in solution. Carbonate hardness is represented by the carbonate and bicarbonate salts of calcium and magnesium. Noncarbonate hardness is represented by all other salts of calcium and magnesium. Hardness is expressed conventionally in terms of an equivalent quantity of calcium carbonate. The following scale may assist the reader in appraising hardness:

$\begin{aligned} & \text { Degree of hardness } \\ & \text { Soft } \\ & \text { Moderately hard }\end{aligned}$
Hard
Very hard

Milligrams per liter (mg/l). A unit for expressing the concentration of chemical c?nstituents by weight per unit of volume. It is numerically equal to parts per mill:on (ppm) in the analyses used in this publication.

pH. A measure of the hydrogen-ion concentration of a solution. A pH unit is expressed as the negative $\log _{10}$ of the hydrogen-ion concentration. The $\mathrm{pH}$ of pure water is 7; acid water has a smaller $\mathrm{pH}$ and alkaline water a larger $\mathrm{pH}$.

Specific conductance. A measure of the ability of a water to conduct an electrical current. It is expressed in micromhos per centimeter at $25^{\circ} \mathrm{C}$. Pure water has a very small electrical conductance, but the conductance increases with increasing concentration of dissolved minerals. 


\title{
CHEMICAL QUALITY OF MICHIGAN STREAMS
}

\author{
By WARREN W. WOOD
}

\begin{abstract}
Concentrations of chemical constituents of Michigan streams exhibit regional patterns that are primarily a function of geology and evapotranspiration. However, in some areas waste disposal by municipal and industrial organizations has altered the natural distribution and concentrations of dissolved material. Concentration and areal distribution of chemical constitutents were found to change very little from high spring to low summer flow conditions.
\end{abstract}

\section{INTRODUCTION}

Chemical quality of streams in Michigan is becoming a matter of increasing public interest and concern. With this public awareness, there is an increase in demand for water quality information by representatives of industry, recreation, and local units of government. This report is intended to provide a source of chemical quality information for these groups and also to present information for statewide comparison and water management planning.

Chemical analyses of samples collected from streams have been published by the U.S. Geological Survey (1943-65) in a series of watersupply papers. These analyses were made from samples collected for specific areal projects as well as those collected from miscellaneous sites throughout the State. Detailed investigations conducted by the Michigan Water Resources Commission also are a source of water quality information on selected areas. (See "Selected references.") However, for this report it was deemed desirable to gather data over the entire State from all types of streams under conditions which are likely to indicate the normal range in chemical quality.

To aid in defining the chemical quality of stream water, samples were collected at arproximately 200 gaging stations (fig. 1). One series of samples was collected in late March and early April 1967 when streamflow was high. Te second set was collected in late August and early September 1967 under conditions of low streamflow.

It is recognized that the hydrologic distribution of gaging stations is not ideal for this type of sampling because many tributaries and several major rivers are not represented. However, the advantages of having instantaneous discharge in addition to long periods of discharge records covering a relatively larg? number of areally well-distributed sample sites is considered to compensate for the lack of a complete sample coverage.

It was intended that analysis of these samples would (1) give comparable values over the entire area of the State under conditions which produce extremes in natural water quality, (2) give regional variation patterns in chemical quality so that reasonable estimates of water quality could be made in the absence of specific analyses, (3) give values to which future measurements may be compared for evaluating changes in water quality, and (4) give a framework on which future and c'otailed water-quality studies can be conducted.

\section{ACKNOWLEDGMENTS}

This project was conducted in cooporation with the Michigan Department of Natural Resources, formerly Department of Conservation, R. A. MacMullan, Director, and under an overall agreement for water resources investigations in Michigan with the State Water Resources 


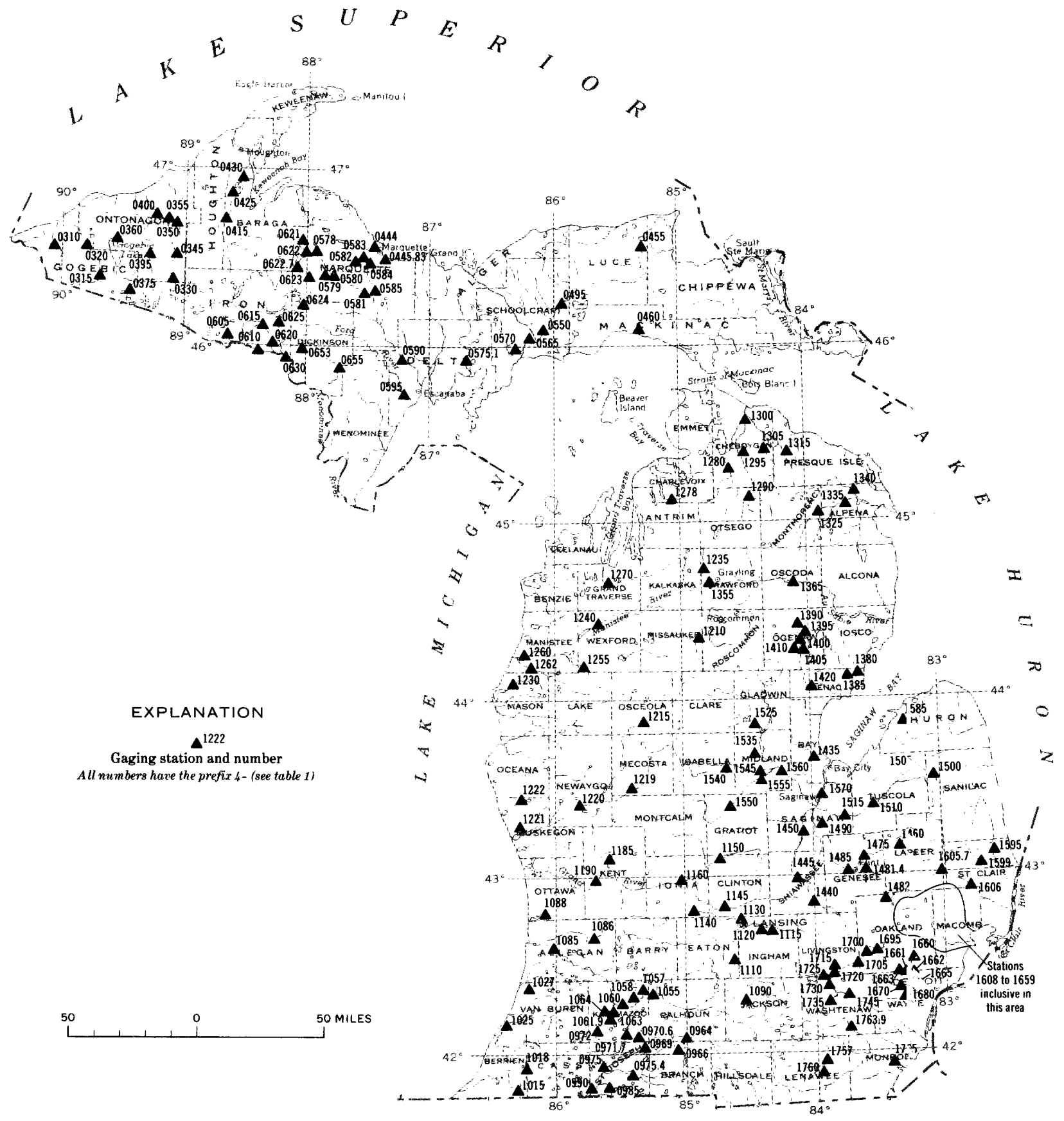

Figure 1.-Locations and identification numbers of sampling sites. 
Commission, Loring F. Oeming, Executive Secretary, succeeded by Ralph W. Purdy.

\section{STREAM QUALITY OBSERVATIONS}

\section{FIELD METHODS OF ANALYSIS}

Samples of water from the stream were collected by personnel of the Michigan District of the U.S. Geological Survey during their regular visits to the gaging stations. The samples were collected in disposable plastic bottles and returned to the district office where chemical analyses were performed using field methods. The time between collection and analysis averaged about 2 weeks. To verify the stability of the parameters during transportation and storage, multiple samples were collected at several sites and analyzed at time intervals up to 1 month in length. There were no changes in the measured constituents greater than those expected from analytical error.

Field methods of analysis consisted of using mercuric nitrate for chloride determination (American Public Health Association, 1965) turbidimetric method for sulfate, colorimetric EDTA (ethylenediaminetetraacetic acid) for hardness, colorimetric and potentiometric titration for bicarbonate, and a conductivity meter (accuracy within 2 percent) for specific conductance. A glass electrode and $\mathrm{pH}$ meter were used to determine $\mathrm{pH}$. Dissolved solids were determined by multiplying specific conductance by 0.65. Results obtained from field methods were within 5 percent of the values obtained from laboratory analyses of 40 duplicate samples.

\section{BASIC QUALITY OF STREAMS}

Figures 2 and 3 illustrate the distribution of the concentration of dissolved solids during periods of low and high flow, respectively. During high-flow conditions streams generally exhibit more dilute water due to the additions of large quantities of rain and snowmelt water. However, the change in concentration between extreme flow conditions is relatively small.

Hardness of stream water (figs. 4, 5) follows the same general pattern as the dissolved solids because the stream water is of the calcium magnesium bicarbonate type. Chloride (figs. 6, 7) and sulfate concentrations (figs. 8, 9) also follow the same distribution pattern. The chlo- ride content in most streams is generally lower than sulfate by a factor of about four. The sulfate ion is usually associated with calcium and magnesium which contribute to the hardness of the water. Chloride results from the presence of dissolved salts of calcium and sodium chloride.

It is apparent from figures 2 through 9 that the mapped chemical constituents increase in concentration from north to south in Michigan streams. Stream water in the western half of the northern peninsula contains the lowest concentrations of dissolved solids, whereas the southeastern section of the southern peninsula contains the highest concentrations. A sampling bias does however exist, because some stream gaging stations are located downstream from significant or unusual waste-water discharges. This bias is particularly noticeable in the Saginaw River system where industrial discharge, rather than natural controls, increased the chloride concentration of the stream. This condition is also apparent in the Grand, Kalamazoo, and Rouge River systems.

The distribution patterns of major concentrations are controlled by differences in bedrock geology, thickness and composition of glacial drift, runoff, and evapotranspiration. Rocks of Precambrian age near the surface in the western half of the northern peninsula (Martin, 1936) are generally very siliceous in composition and would yield small amounts of soluble material to the streams. Because the lithology of the overlying glacial drift is influenced by the underlying bedrock lithology, the drift also yields small amounts of dissolved solids in this area. In the remainder of the State glacial erosion of the Paleozoic and Mesozoic age carbonaceous and argillaceous sediments has contributed fresh unweathered material to the glacial drift. This material is readily soluble and consequently yields significant amounts of dissolved solids to the streams.

Paleozoic age rocks locally contain highly mineralized water (Feth, 1965). Where these rocks are near the surface, they can contribute relatively large amounts of dissolved solids to the stream. This condition is illustrated in the southeastern section of the southern peninsula.

Another factor contributing to the concentration distribution is the greater runoff in the northern part of the State (fig. 10). The difference in runoff is chiefly the result of smaller 


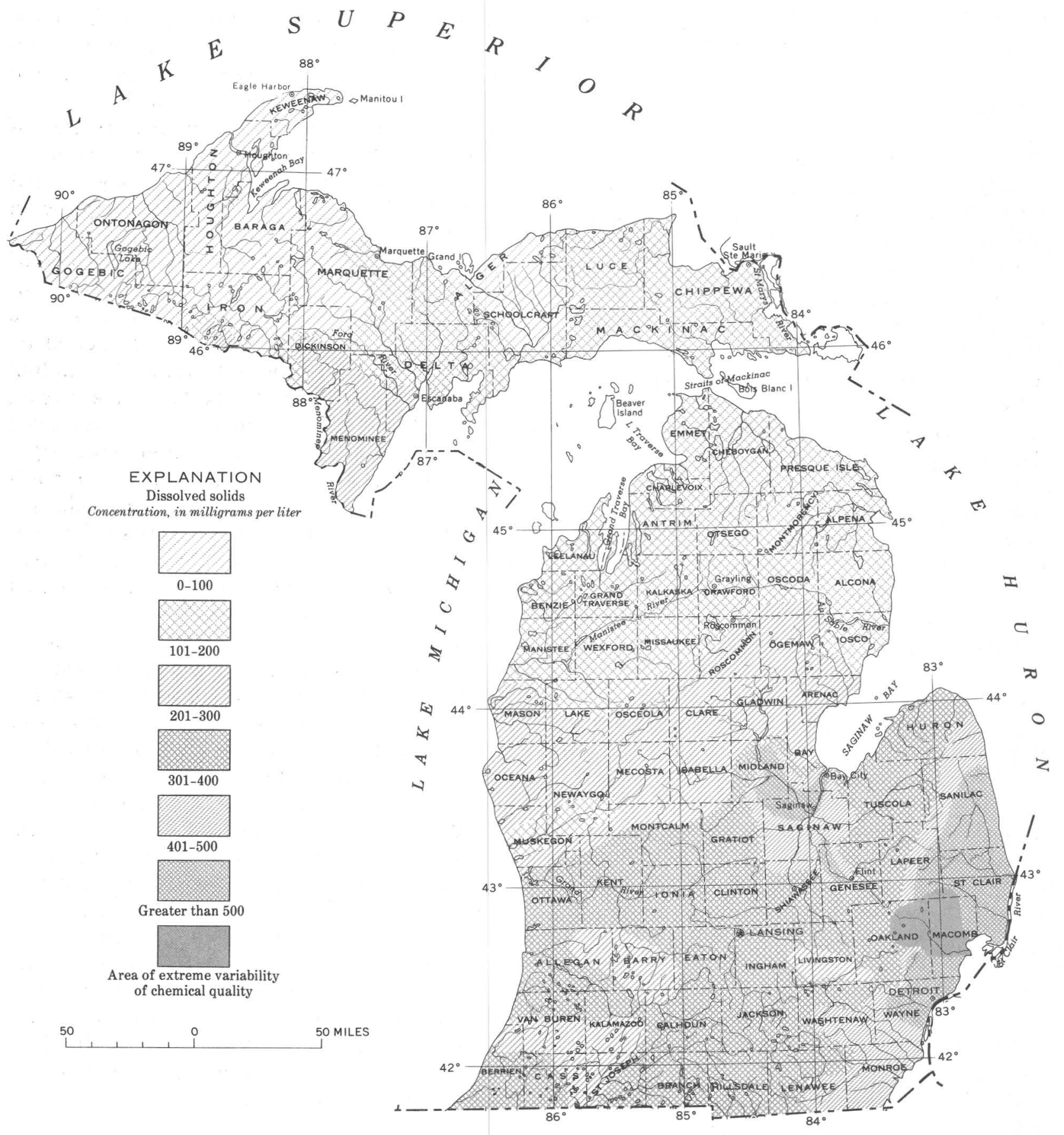

FIGURE 2.-Concentration of dissolved solids in streams under low-flow conditions. 


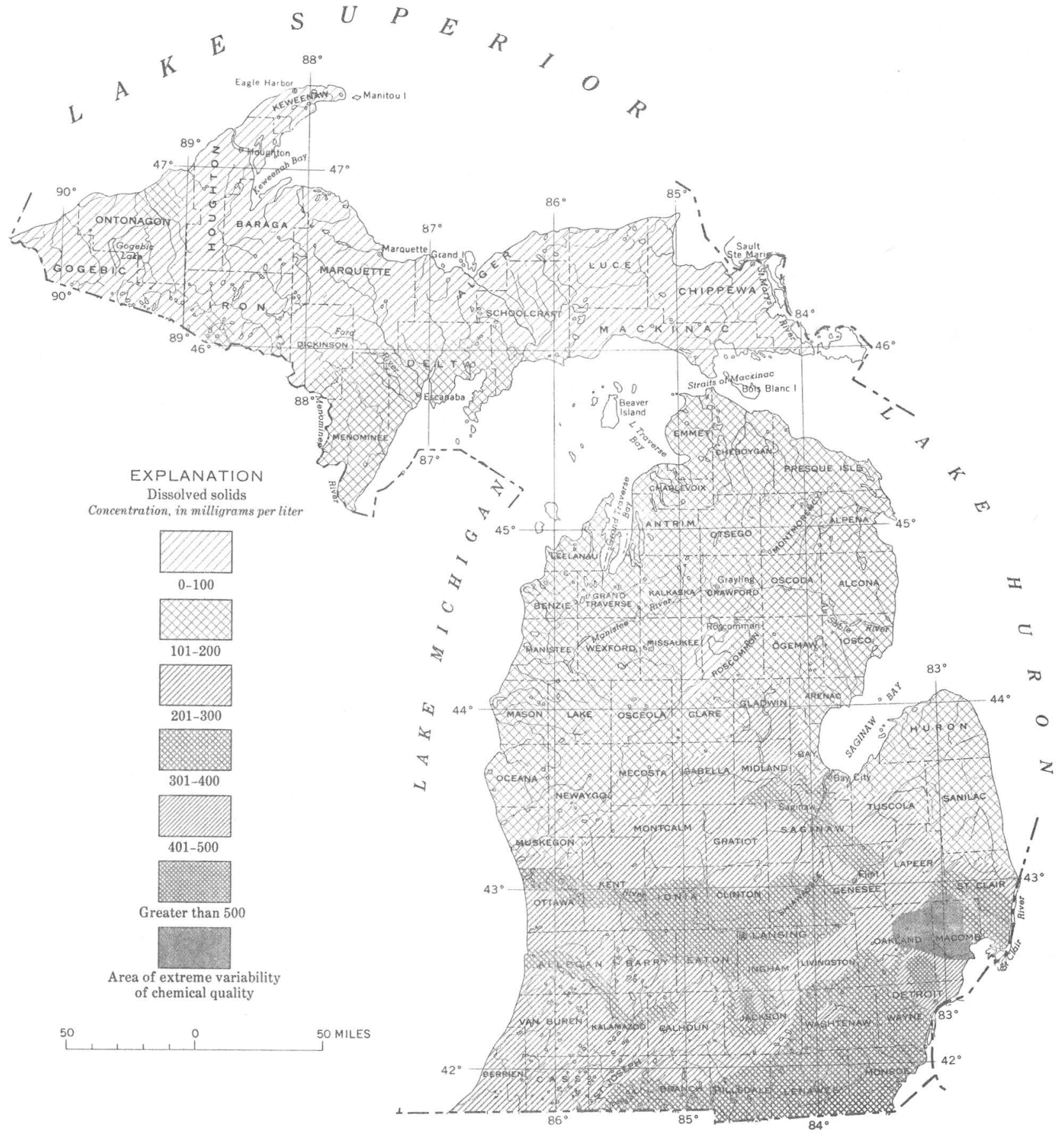

Figure 3.- Concentration of dissolved solids in streams under high-flow conditions. 


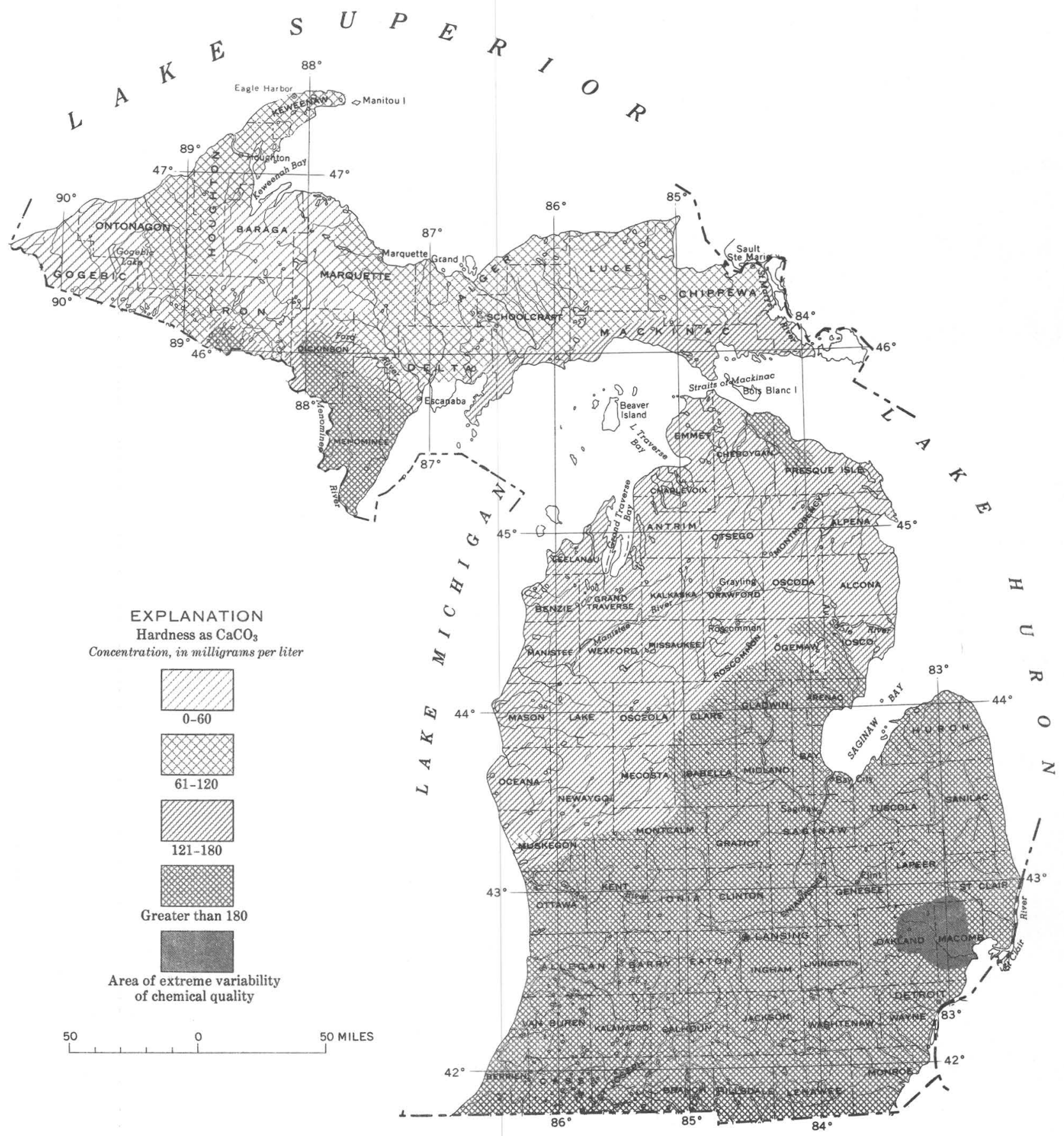

FIGURE 4.-Concentration of hardness of stream water under low-flow conditions. 


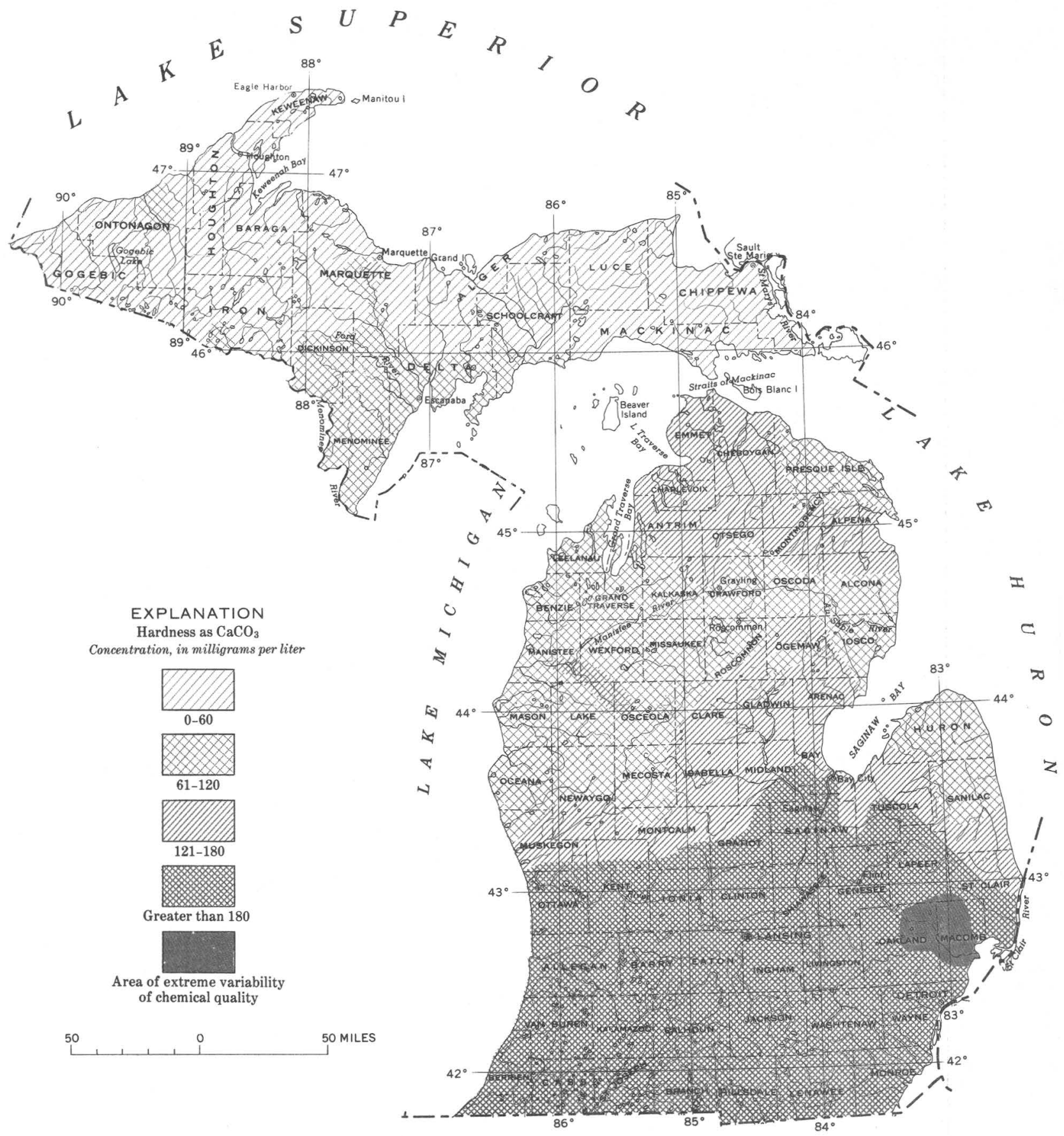

FiguRE 5.-Concentration of hardness of stream water under high-flow conditions. 


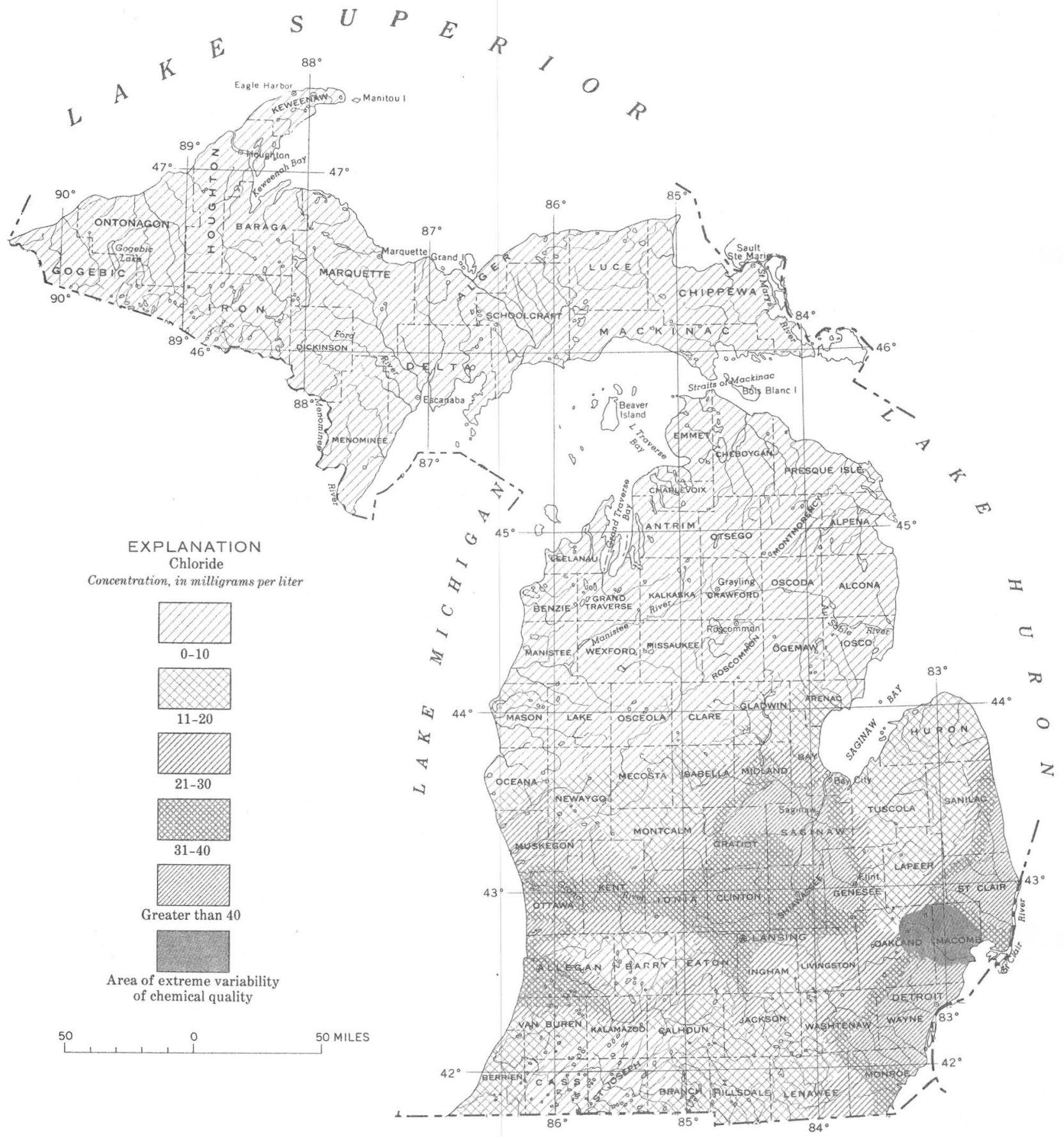

Figure 6.-Concentration of chloride in streams under low-flow conditions. 


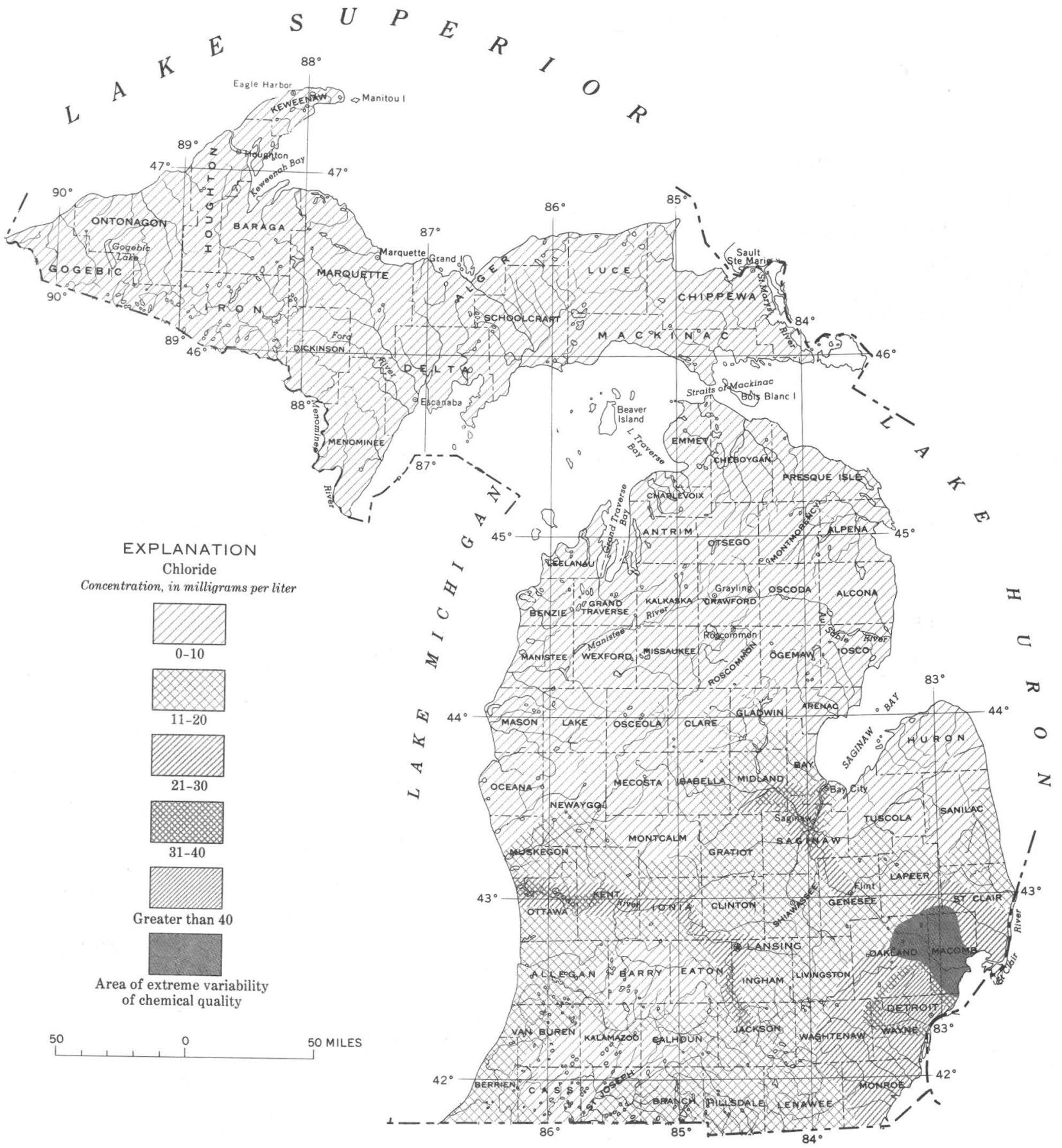

FIGURE 7.-Concentration of chloride in streams under high-flow conditions. 


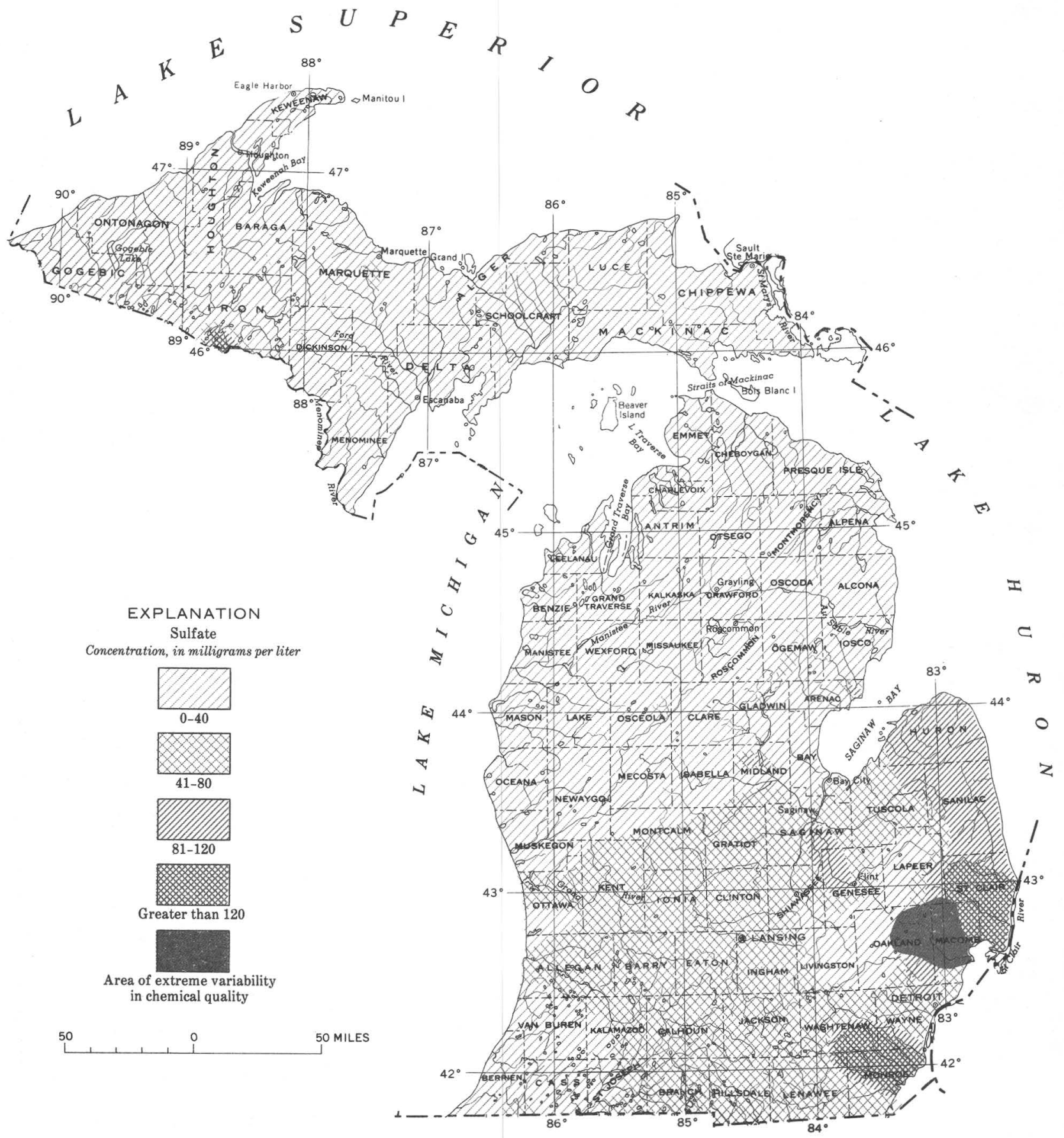

Figure 8.-Concentration of sulfate in streams under low-flow conditions. 


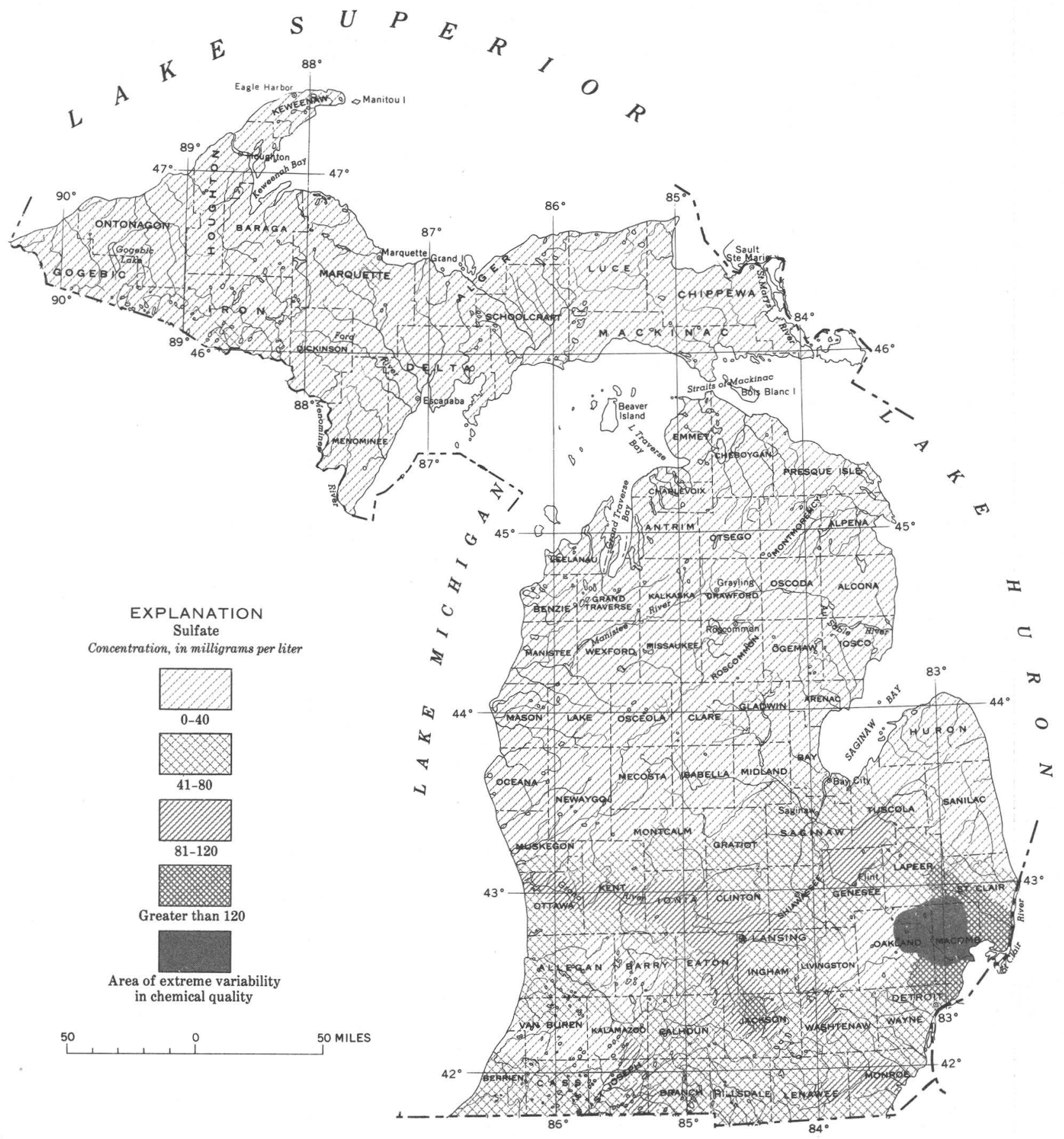

FigURE 9.-Concentration of sulfate in streams under high-flow conditions. 


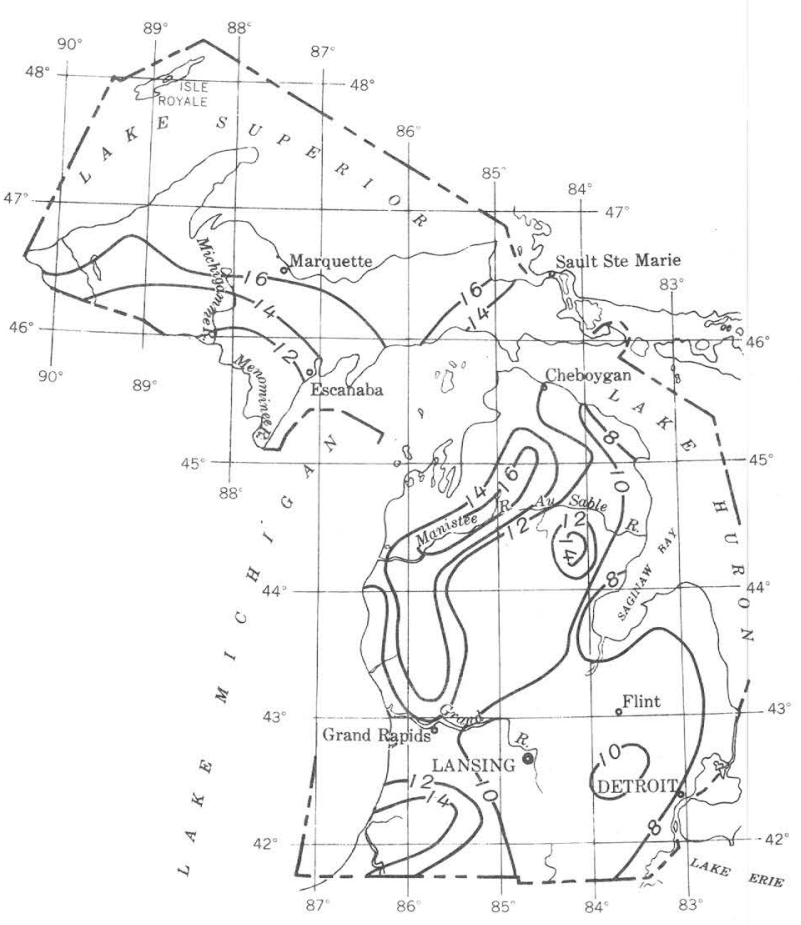

100 MILES

FIGURE 10.-Average annual runoff, in inches, 1951-60.

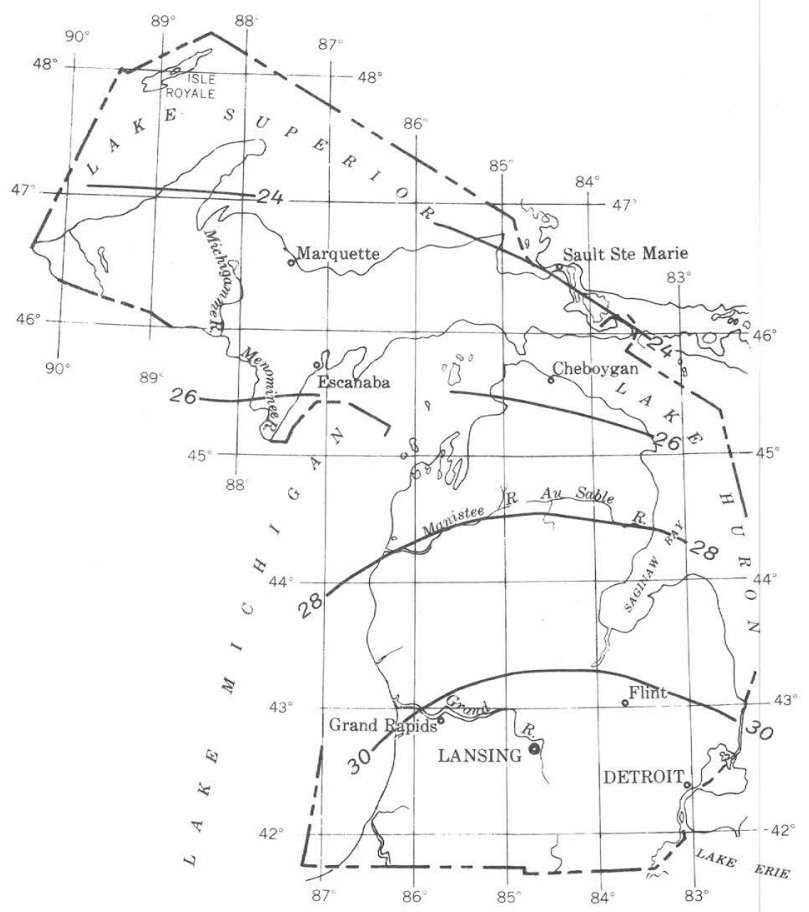

100 MILES

FIgURE 11.-Average annual lake evaporation, in inches, 1946-55.

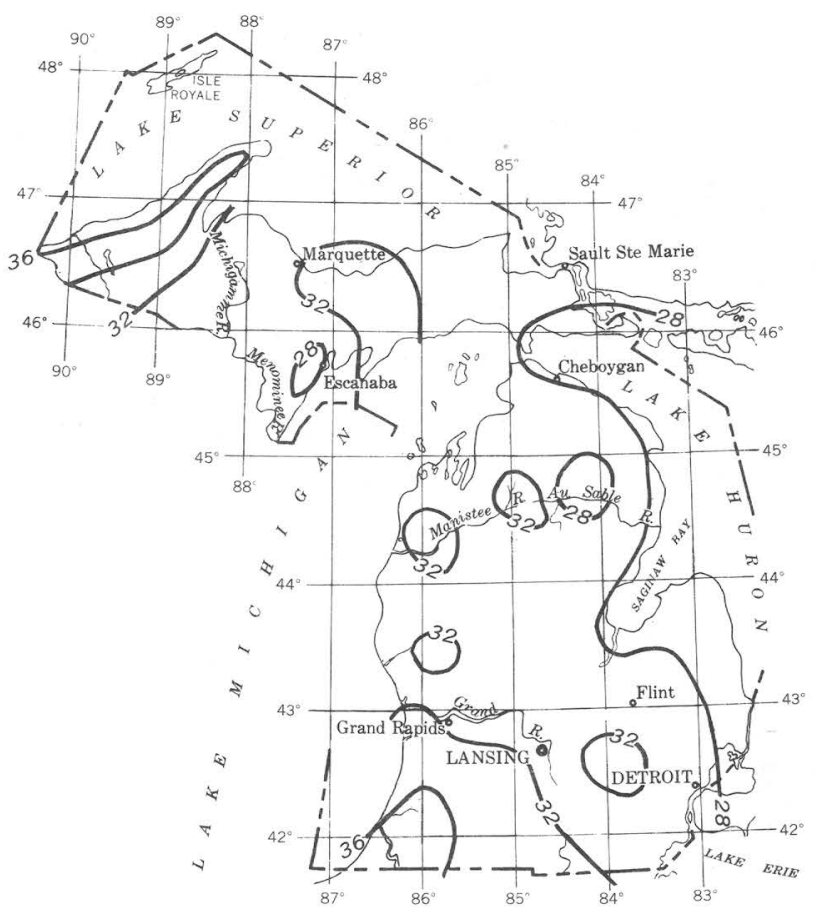

100 MILES

Figure 12.-Mean annual precipitation, in inches 1931-55.

amount of evapotranspiration in the north (fig. 11) (Kohler and others, 1959) rather than greater precipitation (fig. 12) (Strommen, 1967).

In areas of urbanization the concentration of dissolved constituents varies as much as three orders of magnitude within a small stream segment. The dissolved materials come from combination sewers, septic tank effluents, waste treatment from municipal and industrial sources, and the use of soluble components for ice and dust control. Thus, the regional patterns of distribution and concentration are disrupted and the prediction of chemical quality is difficult. Records for the Clinton River basin, station 41608 to 4-1655 (table 1), illustrate the large variation in chemical quality occurring in an urbanized environment.

\section{CONGLUSIONS}

All measured parameters illustrate distinct regional patterns of distribution that shift only slightly under conditions of high and low flow. Water in the streams is of the calcium magnesium bicarbonate type and remains this type 
under high- and low-flow conditions. Concentrations of dissolved substances are generally lower during periods of high flow than during periods of low flow. The higher concentrations that occasionally occur at spring high flows are attributed to inflow of water from combination of eflluent from sewers, fertilizers, and possibly road salt. Considering ali parameters, there is a distinct tendency for the most dilute water to appear in the northern peninsula.

\section{SELECTED REFERENCES}

American Public Health Association, 1965, Standard methods for the examination of water and wastewater: New York, Am. Public Health Assoc., p. 87-89.

Feth, J. H., and others, 1965, Preliminary map of the conterminous United States showing depth to and quality of shallowest ground water containing more than 1,000 parts per million dissolved solids: U.S. Geol. Survey Hydrol. Inv. Atlas HA-199.

Kohler, M. A., Nordenson, T. J., and Baker, D. R., 1959, Evaporation maps for the United States: U.S. Weather Bur. Tech. Paper 37, 13 p.

Martin, H. M., compiler, 1936, The centennial geological map of the northern peninsula of Michigan (and) The centennial geological map of the southern peninsula of Michigan: Michigan Dept. Conserv., Geol. Survey Div. Pub. 39, Geol. Ser. 33.

Michigan Water Resources Commission, 1953, Water resources of the Clinton River basin: Michigan Water Resources Comm., $55 \mathrm{p}$.

- 1955, Water resource conditions and uses in the Paw Paw River basin [revised rept. in 1964]: Michigan Water Resources Comm., 56 p.

- 1956, Water resource conditions and uses in the Flint River basin: Michigan Water Resources Comm., $71 \mathrm{p}$.

1957, Water resource conditions and uses in the Huron River basin: Michigan Water Resources Comm., 149 p.
1960, Water resource conditions and uses in the Tittabawassee River basin: Michigan Water Resources Comm., 117 p.

- 1961, Water resource conditions and uses in the Upper Grand River basin: Michigan Water Resources Comm., $137 \mathrm{p}$.

1963, Water resource conditions and uses in the Shiawassee River basin: Michigan Water Resources Comm., 113 p.

- 1964, Water resource conditions and uses in the Maumee River basin: Michigan Water Resources Comm., $66 \mathrm{p}$.

- 1965, Water resource conditions and uses in the River Raisin basin: Michigan-Water Resources Comm., 105 p.

- 1966, Water resource conditions and uses in the $\mathrm{Au}$ Sable River basin: Michigan Water Resources Comm., 138 p.

- 1967, Water resource conditions and uses in the Lower Grand River basin: Michigan Water Resources Comm., 133 p.

1968, Water resources of southeastern Michigan: Michigan Water Resources Comm., 162 p.

- 1968, Water resources of the Lower Lake Huron drainage basin: Michigan Water Resources Comm., $189 \mathrm{p}$.

Strommen, N. D., 1967, Climates of the states, Michigan: U.S. Dept. Commerce, Climatography of the United States No. 60-20, 25 p.

U.S. Geological Survey, 1943-53, Quality of surface waters of the United States [water years 194149] : U.S. Geol. Survey Water-Supply Papers 942 (1941), 950 (1942), 970 (1943), 1022 (1944), 1030 (1945), 1050 (1946), 1102 (1947), 1132 (1948), and $1162(1949)$.

-1954-65, Quality of surface waters of the United States, Parts 3 and 4, Ohio River basin and St. Lawrence River basin [water years 1950-63]: U.S. Geol. Survey Water-Supply Papers 1186 (1950), 1197 (1951), 1250 (1952), 1290 (1953), 1350 (1954), $1400(1955), 1450$ (1956), 1520 (1957), 1571 (1958), 1642 (1959), 1742 (1960), 1882 (1961), 1942 (1962), 1948 (1963), and 1957 (Part 4, 1964-65). 
TABLE 1 
TABLE 1.-Chemical analyses of samples collected from Michigan streams under low-and high-flow conditions, 1967

[Chemical analyses of field-measured samples, in milligrams per liter]

\begin{tabular}{|c|c|c|c|c|c|c|c|c|c|c|c|c|c|c|c|c|}
\hline \multirow{3}{*}{$\begin{array}{l}\text { Station } \\
\text { No. }\end{array}$} & \multirow{3}{*}{ Station Name } & \multirow{3}{*}{ Location } & \multirow{3}{*}{$\begin{array}{l}\text { Drain. } \\
\text { age } \\
\text { area } \\
\text { (sq mi) }\end{array}$} & \multirow{3}{*}{$\begin{array}{c}\text { Date } \\
\text { sampled }\end{array}$} & \multirow{3}{*}{ Time } & \multirow{3}{*}{$\begin{array}{l}\text { Dis- } \\
\text { charge } \\
\text { (cfs) }\end{array}$} & \multirow{3}{*}{$\begin{array}{l}\text { Bicar- } \\
\text { bonate } \\
\left(\mathrm{HCO}_{3}\right)\end{array}$} & \multirow{3}{*}{$\begin{array}{c}\text { Car- } \\
\text { bon- } \\
\text { ate } \\
\text { (CO })\end{array}$} & \multirow{3}{*}{$\begin{array}{r}\text { Sul- } \\
\text { fate } \\
\text { (S00.) }\end{array}$} & \multirow{3}{*}{$\begin{array}{l}\text { Chlo- } \\
\text { ride } \\
\text { (Cl) }\end{array}$} & \multicolumn{2}{|c|}{$\begin{array}{c}\text { Hardness } \\
\text { as CaCO. }\end{array}$} & \multirow{3}{*}{$\begin{array}{c}\text { Specific } \\
\text { conduc- } \\
\text { tance } \\
\text { (micro- } \\
\text { mhos at } \\
25^{\circ} \mathrm{C} \text { ) }\end{array}$} & \multirow{3}{*}{$\mathrm{pH}$} & \multirow{2}{*}{\multicolumn{2}{|c|}{$\begin{array}{c}\text { Water } \\
\text { temperature }\end{array}$}} \\
\hline & & & & & & & & & & & \multirow{2}{*}{$\begin{array}{l}\mathrm{Ca}, \\
\mathrm{Mg},\end{array}$} & \multirow{2}{*}{$\begin{array}{l}\text { Non- } \\
\text { car- } \\
\text { bon- } \\
\text { ate } \\
\end{array}$} & & & & \\
\hline & & & & & & & & & & & & & & & $F^{\circ}$ & $\mathrm{C}^{2}$ \\
\hline \multicolumn{17}{|c|}{ STREAMS TRIBUTARY TO LAKE SUPERIOR } \\
\hline $4-0310 \ldots \ldots$ & $\begin{array}{l}\text { Black River near } \\
\text { Bessemer. }\end{array}$ & SEY $/ 4 \sec .32$, T. 48 N., R.46 W. & 200 & $\begin{array}{l}\text { Apr } 1 \\
\text { Sept. } 6\end{array}$ & 1030 & 3000 & 20 & 0 & 3.0 & 2.0 & 20 & 4 & 55 & 7.4 & 33 & 0.5 \\
\hline $0315 \ldots \ldots$ & $\begin{array}{l}\text { Presque Isle River at } \\
\text { Marenisco. }\end{array}$ & NW1/4ec. 21, T. 46 N., R.43 W. & 171 & $\begin{array}{l}\text { Apt. } \\
\text { Apr. } 11 \\
\text { Sept. } 8\end{array}$ & $\begin{array}{l}9950 \\
1150 \\
11220\end{array}$ & $\begin{array}{r}50.7 \\
855\end{array}$ & ${ }_{28}^{56}$ & 8 & 2.0 & $\begin{array}{l}5.0 \\
10\end{array}$ & 54 & 8 & $\frac{105}{60}$ & 7.4 & $\begin{array}{l}60 \\
35\end{array}$ & \\
\hline $0320 \ldots \ldots$ & $\begin{array}{l}\text { Presque Isle River near } \\
\text { Tulsa }\end{array}$ & NW1/48ec. 23, T. 48 N., R.44 W. & 261 & $\begin{array}{l}\text { Apr. } 10^{*} \\
\text { Sept. } 7\end{array}$ & $\begin{array}{l}1400 \\
10150 \\
1015\end{array}$ & $\begin{array}{l}80.0 \\
1730\end{array}$ & $\begin{array}{l}54 \\
15\end{array}$ & $\begin{array}{l}0 \\
0\end{array}$ & $\begin{array}{l}2.0 \\
10^{2}\end{array}$ & $\begin{array}{l}2.00 \\
2.0\end{array}$ & $\begin{array}{l}20 \\
48 \\
18\end{array}$ & $\begin{array}{l}4 \\
6\end{array}$ & $\begin{array}{l}80 \\
38\end{array}$ & $\begin{array}{l}7.1 \\
7.0\end{array}$ & $\begin{array}{l}65 \\
63 \\
28\end{array}$ & $\begin{array}{r}18.5 \\
3.5 \\
170\end{array}$ \\
\hline $\begin{array}{l}0330 \ldots \ldots \\
0345 \ldots \ldots\end{array}$ & $\begin{array}{l}\text { Middle Branch Onton- } \\
\text { agon near Paulding. } \\
\text { Middle Branch onton. } \\
\text { agon River near Trout } \\
\text { Creek. }\end{array}$ & $\begin{array}{l}\text { NE1/sec. } 29 \text { T. } 46 \text { N., R.38 W. } \\
\text { SW1/4sec. 8, T. } 47 \text { N., R.38 W. }\end{array}$ & $\begin{array}{l}164 \\
203\end{array}$ & $\begin{array}{l}\text { Apr. 2 } \\
\text { Sept. } \\
\text { Sept. } 7\end{array}$ & $\begin{array}{l}0930 \\
1110 \\
2015\end{array}$ & $\begin{array}{l}117 \\
590 \\
116 \\
435\end{array}$ & $\begin{array}{l}56 \\
38 \\
88 \\
76\end{array}$ & $\begin{array}{l}0 \\
0 \\
0 \\
0\end{array}$ & $\begin{array}{l}1.0 \\
4.0 \\
8.0 \\
7.0\end{array}$ & $\begin{array}{l}2.0 \\
2.0 \\
2.0 \\
2.0\end{array}$ & $\begin{array}{l}50 \\
32 \\
74 \\
63\end{array}$ & $\begin{array}{l}4 \\
1 \\
1 \\
1\end{array}$ & $\begin{array}{r}100 \\
75 \\
150 \\
125\end{array}$ & $\begin{array}{l}7.3 \\
7.6 \\
7.3 \\
7.3\end{array}$ & $\begin{array}{l}63 \\
33 \\
61 \\
60\end{array}$ & $\begin{array}{l}17.0 \\
16.0 \\
15.5\end{array}$ \\
\hline $0350 \ldots \ldots$ & $\begin{array}{l}\text { East Branch Ontonagon } \\
\text { Biver near Masg }\end{array}$ & NW3/48ec. 33, T. 50 N., R.38 W. & 272 & Mar. 31 & 1630 & 2920 & 83 & 0 & 20 & 2.0 & 68 & 0 & 135 & 7.6 & 32 & $\begin{array}{r}0.0 \\
16.5\end{array}$ \\
\hline $0355 \ldots \ldots . .$. & $\begin{array}{l}\text { Middle Branch Onton. } \\
\text { agon River near } \\
\text { Rockland }\end{array}$ & SE1/4sec. 27, T. 50 N., R.39 W. & 671 & Mar. 31 & 1535 & 9190 & 104 & 0 & $11^{1.0}$ & 2.0 & 83 & 0 & $\begin{array}{l}160 \\
160\end{array}$ & 7.8 & 32 & 0.0 \\
\hline $0360 \ldots \ldots$ & $\begin{array}{l}\text { West Branch Ontonagon } \\
\text { Wer }\end{array}$ & $\mathrm{NE3} / 4 \mathrm{sec} .3$, T. $48 \mathrm{~N} ., \mathrm{R} .42 \mathrm{~W}$. & 162 & Apr. 10 & 1545 & 530 & 26 & 0 & 5.0 & 2.0 & 25 & 4 & 60 & 7.4 & 36 & 2.0 \\
\hline 0375 ...... & $\begin{array}{l}\text { Cisco Branch Ontoongog } \\
\text { River at Cisco Lake } \\
\text { Outlet. }\end{array}$ & $\mathrm{E} 1 / 2 \sec .32$, T. 45 N., R.41 W. & 50.7 & $\begin{array}{l}\text { Apr. } 11 \\
\text { Aug. } 2\end{array}$ & $\begin{array}{l}1110 \\
1315 \\
0955 \\
1730\end{array}$ & $\begin{array}{l}199 \\
12.2\end{array}$ & $\begin{array}{l}52 \\
51 \\
51\end{array}$ & $\begin{array}{l}0 \\
0 \\
0\end{array}$ & $\begin{array}{l}17.0 \\
5.5 \\
6.5\end{array}$ & $\begin{array}{l}4.0 \\
2.0 \\
2.0\end{array}$ & $\begin{array}{l}28 \\
46 \\
42\end{array}$ & $\begin{array}{l}4 \\
0 \\
0\end{array}$ & $\begin{array}{r}60 \\
100 \\
85\end{array}$ & $\begin{array}{l}7.0 \\
7.7 \\
7.4\end{array}$ & $\begin{array}{l}67 \\
40 \\
71\end{array}$ & $\begin{array}{r}1.5 \\
4.5 \\
21.5\end{array}$ \\
\hline 0395 & $\begin{array}{l}\text { South Branch Ontonagon } \\
\text { River at Ewen. }\end{array}$ & NW1/4sec. 26, T., 48 N., R.40 W. & 348 & $\begin{array}{l}\text { A pre. } \\
\text { Sept. } 7 \\
\text { S. }\end{array}$ & $\begin{array}{l}11300 \\
1800\end{array}$ & $\begin{array}{r}350 \\
16900\end{array}$ & $\begin{array}{l}15 \\
68 \\
88\end{array}$ & $\begin{array}{l}n \\
0 \\
0\end{array}$ & $\begin{aligned} 1.0 \\
7.0 \\
29\end{aligned}$ & $\begin{array}{r}2.0 \\
4.0 \\
.5\end{array}$ & $\begin{array}{l}18 \\
60 \\
84\end{array}$ & $\begin{array}{r}6 \\
4 \\
12\end{array}$ & $\begin{array}{l}45 \\
120 \\
172\end{array}$ & $\begin{array}{l}7.5 \\
7.3 \\
8.0\end{array}$ & $\begin{array}{l}33 \\
62 \\
32\end{array}$ & $\begin{array}{r}0.5 \\
16.5 \\
0.0\end{array}$ \\
\hline $\begin{array}{l}0400 \ldots . \\
0415 \ldots\end{array}$ & $\begin{array}{l}\text { Ontongagon River near } \\
\text { Rockland. } \\
\text { Sturgeon River near }\end{array}$ & $\begin{array}{l}\mathrm{NE} 2 / 4 \mathrm{sec}, 20, \text { T. } 50 \mathrm{~N} ., \mathrm{R} .39 \mathrm{~W} . \\
\mathrm{SE1} / \mathrm{sec} .15, \mathrm{~T}, 50 \text { N., R.35 W. }\end{array}$ & $\begin{array}{r}1340 \\
346\end{array}$ & $\begin{array}{l}\text { Mart. } 29 . \\
\text { Sept. } 21 \\
\text { Aung } 21\end{array}$ & $\begin{array}{l}1430 \\
1515\end{array}$ & $\begin{array}{l}560 \\
600\end{array}$ & $\begin{array}{l}80 \\
51\end{array}$ & $\begin{array}{l}0 \\
0\end{array}$ & $\begin{array}{l}\mathbf{5 . 0} \\
3.0\end{array}$ & $\begin{array}{l}3.0 \\
3.0\end{array}$ & $\begin{array}{l}66 \\
46\end{array}$ & ${ }_{4}^{0}$ & $\begin{array}{r}140 \\
80\end{array}$ & $\begin{array}{l}7.2 \\
7.3\end{array}$ & $\begin{array}{l}52 \\
62\end{array}$ & $\begin{array}{l}11.0 \\
16.5\end{array}$ \\
\hline $0425 \ldots \ldots$ & $\begin{array}{l}\text { Aston. } \\
\text { Otter River near Elo. }\end{array}$ & $\mathrm{NE} / 4 \mathrm{sec} .8$, T. 50 N., R.34 W. & 162 & Apr. 4 & 1000 & 499 & 39 & 0 & 5.0 & 2.0 & 36 & 4 & 85 & 7.4 & 34 & 1.0 \\
\hline $\begin{array}{l}0430 \ldots \ldots \\
0444 \ldots \ldots\end{array}$ & $\begin{array}{l}\text { Sturgeon River near } \\
\text { Arnheim. } \\
\text { Carp River near }\end{array}$ & $\begin{array}{l}\text { SEY/4ee. 1, T. } 52 \text { N., R.34 W. } \\
\text { SE1/8ec. 29, T. } 48 \text { N., R.26 W. }\end{array}$ & $\begin{array}{l}705 \\
51.4\end{array}$ & $\begin{array}{l}\text { Aug. } 21 \\
\text { Apr. } 4^{*} \\
\text { Aug. } 23 \\
\text { Aug. } 16\end{array}$ & $\begin{array}{l}1600 \\
1030 \\
1625\end{array}$ & $\begin{array}{c}84.2 \\
3790 \\
401 \\
35.7\end{array}$ & $\begin{array}{r}112 \\
33 \\
76 \\
98\end{array}$ & $\begin{array}{l}0 \\
0 \\
0 \\
0\end{array}$ & $\begin{array}{r}6.0 \\
9.6 \\
2.0 \\
13\end{array}$ & $\begin{array}{l}4.0 \\
2.0 \\
3.0 \\
8.0\end{array}$ & $\begin{array}{l}88 \\
32 \\
64 \\
92\end{array}$ & $\begin{array}{r}0 \\
5 \\
2 \\
12\end{array}$ & $\begin{array}{l}180 \\
73 \\
125 \\
190\end{array}$ & $\begin{array}{l}7.8 \\
77.0 \\
7.4\end{array}$ & $\begin{array}{l}57 \\
32 \\
63 \\
73\end{array}$ & $\begin{array}{r}14.0 \\
00 \\
17.0 \\
230\end{array}$ \\
\hline $\begin{array}{l}0445.83 . \\
0455 \ldots \ldots\end{array}$ & $\begin{array}{l}\text { Negaunee. } \\
\text { Therry Creek near Harvey. } \\
\text { Tahquamenon River near } \\
\text { Tahquamenon Paradise. }\end{array}$ & $\begin{array}{l}\mathrm{SE3} / \mathrm{sec}, 13, \mathrm{~T}, 47 \mathrm{~N}, \mathrm{R} .25 \mathrm{~W} \\
\mathrm{NE} / 4 \mathrm{sec} .11, \mathrm{~T} .48 \mathrm{~N}, \mathrm{R} .8 \mathrm{~W}\end{array}$ & 790.53 & $\begin{array}{l}\text { Aug. } 16 \\
\text { Sept. } 7\end{array}$ & $\begin{array}{l}1900 \\
1200\end{array}$ & $\begin{array}{c}18.2 \\
293\end{array}$ & $\begin{array}{l}107 \\
105\end{array}$ & $\begin{array}{l}0 \\
0\end{array}$ & ${ }_{14}^{6.0}$ & $\begin{array}{l}4.0 \\
3.0\end{array}$ & $\begin{array}{l}92 \\
98\end{array}$ & $\begin{array}{r}4 \\
12\end{array}$ & $\begin{array}{l}180 \\
190\end{array}$ & $\begin{array}{l}77.7 \\
7.3\end{array}$ & $\begin{array}{l}49 \\
67\end{array}$ & $\begin{array}{r}9.5 \\
19.5\end{array}$ \\
\hline
\end{tabular}

\section{STREAMS TRIBUTARY TO LAKE MICHIGAN}

\begin{tabular}{|c|c|}
\hline $\begin{array}{r}4-0460 \ldots \ldots \\
0495 \ldots \ldots\end{array}$ & $\begin{array}{l}\text { Black River near Garnet. } \\
\text { Manistique River at }\end{array}$ \\
\hline $0550 \ldots \ldots$ & Manistique River near \\
\hline $0565 \ldots \ldots$ & $\begin{array}{l}\text { Blaney. } \\
\text { Manistique River near }\end{array}$ \\
\hline $0570 \ldots \ldots$ & $\begin{array}{l}\text { Manistique. } \\
\text { Indian River near }\end{array}$ \\
\hline $0575.1 \ldots$ & $\begin{array}{l}\text { Mansistique. } \\
\text { Sturgeon River near }\end{array}$ \\
\hline $0578 \ldots \ldots . . .$. & $\begin{array}{l}\text { Nanma Junction. } \\
\text { Middle Branch Escanaba }\end{array}$ \\
\hline $0579 \ldots \ldots$ & $\begin{array}{l}\text { River at Humboldt. } \\
\text { Black River near }\end{array}$ \\
\hline $0580 \ldots \ldots$ & $\begin{array}{l}\text { Republhc. } \\
\text { Middle Branch Eesanaba }\end{array}$ \\
\hline $0581 \ldots \ldots$ & $\begin{array}{l}\text { River near Ishpeming. } \\
\text { Middle Branch Escanabe }\end{array}$ \\
\hline $0532 \ldots \ldots$ & $\begin{array}{l}\text { Schweitser Creek near. } \\
\text { Palmer. }\end{array}$ \\
\hline
\end{tabular}
SE1/4sec. $13, \mathrm{~T}, 43 \mathrm{~N} ., \mathrm{R} .9 \mathrm{~W}$.

$\mathrm{SE} / \mathrm{s} / \mathrm{sec}, 28, \mathrm{~T} .43 \mathrm{~N}, \mathrm{R} .14 \mathrm{~W}$ SE, $/ 4$ sec. $15, T .42$ N., R.15 W. NE1/4sec. 34, T. 42 N., R.16 W. SE1/1sec. 17 T. 41 N., R.19 W.

\begin{tabular}{|c|c|}
\hline$\frac{28.0}{341}$ & $\begin{array}{l}\text { Aug. } 16 \\
\text { Apr. } 3\end{array}$ \\
\hline$\cdot 704$ & $\begin{array}{l}\text { Aug. } 17 \\
\text { Apr. } 5\end{array}$ \\
\hline 1100 & $\begin{array}{l}\text { Aug. } 16 \\
\text { Apr. } 5^{*}\end{array}$ \\
\hline 302 & $\begin{array}{l}\text { Aug. } 16 \\
\text { Apr. } 22\end{array}$ \\
\hline $18 ?$ & $\begin{array}{l}\text { Aug. } 16 \\
\text { Ang. } 18\end{array}$ \\
\hline 46 & $A$ \\
\hline 34.4 & Apr. 4 \\
\hline 128 & Apr. 4 \\
\hline 210 & $\begin{array}{l}\text { Aug. } 16 \\
\text { Apr. } 4\end{array}$ \\
\hline 23.6 & $\begin{array}{l}\text { Aug. 14 } \\
\text { Apr. } 3 \\
\text { Aug. 16 }\end{array}$ \\
\hline
\end{tabular}

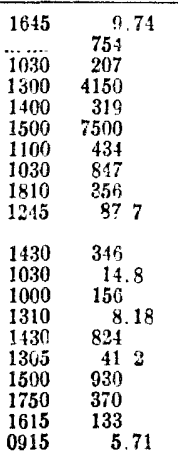

$\begin{array}{rr}0 & 4.0 \\ 0 & 12 \\ 0 & 9.0 \\ 0 & 0 . \\ 0 & 8 \\ 0 & 11 \\ 0 & 8 \\ 0 & 19 \\ 0 & 48 \\ 0 & 9 \\ 0 & 5.0 \\ 0 & 8 . \\ 0 & 6 . \\ 0 & 16 \\ 0 & 11 \\ 0 & 7.0 \\ 0 & 8.0 \\ 0 & 1.0 \\ 0 & 7.0 \\ 0 & 4.0\end{array}$

4.0
12
9.0
6.0
85
11
80
19
48
99
5.0
8.0
6.0
16
11
7.0
8.0
1.0
7.0
4.0

$\begin{array}{rr}2.0 & 13 \\ 1.0 & 3 \\ 1.0 & 6 \\ 2.0 & 2 \\ 20 & 7 \\ 2.0 & 3 \\ 2.0 & 77 \\ 20 & 8 \\ 20 & 13 \\ 2.0 & 0 \\ 1.0 & 15 \\ 4.0 & 4 \\ 4.0 & 2 \\ 60 & 5 \\ 3.0 & 17 \\ 4.0 & 49 \\ 2.0 & 1 \\ 4.0 & 40 \\ \mathbf{3 . 0} & 6 \\ .0 & 47\end{array}$

134
34
66
29
79
30
77
85
132
07
15
40
20
59
17
40
18
40
60
470

$\begin{array}{rrrr}245 & 8.2 & 60 & 15.5 \\ 75 & 7.5 & 35 & 11.5 \\ 130 & 7.1 & 66 & 19.0 \\ 60 & 75 & 33 & 0.5 \\ 140 & 7.5 & 70 & 2100 \\ 66 & 7.4 & 32 & 0.0 \\ 150 & 7.6 & 69 & 20.5 \\ 170 & 7.8 & 36 & 2.0 \\ 255 & 7.8 & 76 & 24.5 \\ 195 & 9.2 & 65 & 10.0 \\ & & \\ <50 & 7.4 & 34 & 1.0 \\ 85 & 7.2 & 58 & 14.5 \\ 55 & 7.1 & 34 & 1.0 \\ 130 & 7.0 & 69 & 20.5 \\ 50 & 7.6 & 34 & 1.0 \\ 80 & 7.3 & 67 & 19.5 \\ 50 & 7.6 & 33 & 0.5 \\ 85 & 7.1 & 68 & 20.0 \\ 135 & 7.6 & 34 & 10 \\ 140 & 7.4 & 54 & 12.0\end{array}$






NW1/4sec. 10, T. 46 N., R.26 W. SE1/4sec. 12 , T. $46 \mathrm{~N}, \mathrm{R} .26 \mathrm{~W}$. NE1/4sec. 21, T. 45 N., R.25 W. NW1/4sec. 32, T. 41 N., R.23 W. SW $1 / 4$ sec. 19, T. 39 N., R.23 W. SW1/4sec. 1, T. 42 N., R.35 W. SE1/4sec. 11, T. 41 N., R.32 W. SE $1 / 4$ sec. 20, T. 43 N., R.32 W. NW/4sec. 25, T. 42 N., R.32 W. SE 14 sec, 1, T. 48 N., R.30 W. NW1/4sec. 13, T. 48 N., R.30W. NW1/4sec. 27, T. 47 N., R.30 W. $\mathrm{SE}^{\mathrm{1}} \mathbf{4} \mathrm{sec} .18, T$ T. 46 N., R.29 W. NW1/4sec. 1, T. 44 N., R.30 W. NW!/sec. 20, T. 43 N., R.31 W. NE $1 / 4$ sec. 16, T. 41 N., R.31 W. NE $1 / 4$ sec. 30, T. 42 N., R.29 W. NW1/4sec. 36 T. 41 N., R.28 W. SW1/4sec. 20, T. 4 S., R.6 W. $\mathrm{N}, 2$ sec. 22, T. 5 S., R.7 W. NW1/4sec. 12, T. 5 \&., R.9 W. SW1/4sec. 29, T. 4 S., R.9 W. SW1/4sec. 16, T. 4 S., R.10 W. NE1/4sec. 5, T. 4 S., R.11 W. S! 2 sec, 18, T. 6 S., R.11 W. SW 1/4sec. 6, T. 7 S., R.9 W. SW1/4sec. 10, T. 8 S., R.11 W. SW1/4sec. 6, T. 8 S., R.12 W. SW1/4sec. 26, T. 7 S., R.17 W. SE)/4sec. 30, T. 6 S., R.16 W. NE1/4sec. 26, T. 3 s., R.18 W. NW1/4sec. 28, T. 1 S., R.16 W. SW1/4sec. 1, T. 2 S., R.8W. SW1/4sec. 27, T. 1 S., R.9 W. NE $1 / 4$ sec. 7, T. 2 S., R.9 W. NE1/4sec. 19, T. 2 S., R.10 W. NW1/4sec. 15, T. 3 S., R.11 W. SE1/4sec. 34, T. 2 S., R.11 W.
$14.2 \mathrm{Apr} .3$ 37.5 Apr. 11 124 Aug. 15 870 Apr. 13* 450 Apr. $13^{*}$ 92.1 Aug. 1* $^{*}$

597 Apr. 11 631 Aug. $11 *$ 66.5 Apr. 10 $\begin{array}{cc}1635 & 764 \\ 2 ? 0\end{array}$ $\begin{array}{llll}133 & \text { Apr. } 10 & 1530 & 1330 \\ & \text { Aug. } 18 & 1450 & 44.9\end{array}$ $231 \quad$ Apr. 18 1115 2680 240 Aug. $18 \quad 1730 \quad 146$

$316 \quad$ Apr. $18 \quad 1300 \quad 2960$

Aug. 18 $\begin{array}{lll}\text { Aug. } 31 \quad 1830 & 616\end{array}$ $\begin{array}{lllr}780 & \text { Apr. } 18 & 1400 & 11200 \\ & 0830 & 545\end{array}$

56.1 Aug. 21 237 Aug. 25

68.2 Mar. 14 7.29 Apr. 12

1350 Aug. 15

106 Aug. 15

192 Aug. 14

$\begin{array}{lll} & 0940 & 21.4 \\ 508 & \\ & 1130 & 65.4\end{array}$ $\begin{array}{lll}\text { Aug. } 15 & 1220 & 4510 \\ \text { Apr. } 8 & 0905 & 513\end{array}$ $\begin{array}{lll} & 0905 & 539\end{array}$ $3666 \quad \begin{array}{llll}\text { A pr. } 8^{*} & 1545 & 7310 \\ 1600 & 1460\end{array}$ 
TABLE 1.-Chemical analyses of samples collected from Michigan streams under low-and high-flow conditions, 1967-Continued

\begin{tabular}{|c|c|c|c|c|c|c|c|c|c|c|c|c|c|c|c|c|}
\hline \multirow{3}{*}{$\begin{array}{c}\text { Station } \\
\text { No. }\end{array}$} & \multirow{3}{*}{ Station Name } & \multirow{3}{*}{ Lncation } & \multirow{3}{*}{$\begin{array}{l}\text { Drain. } \\
\text { age } \\
\text { area } \\
\text { (sy mi) }\end{array}$} & \multirow{3}{*}{$\begin{array}{l}\text { Date } \\
\text { sampled }\end{array}$} & \multirow{3}{*}{ Time } & \multirow{3}{*}{$\begin{array}{c}\begin{array}{c}\text { Dis- } \\
\text { charge } \\
\text { (cfs) }\end{array} \\
\text { (cfs }\end{array}$} & \multirow{3}{*}{$\begin{array}{l}\text { Birar- } \\
\text { bonate } \\
\left(\mathrm{HCO}_{3}\right)\end{array}$} & \multirow{3}{*}{$\begin{array}{l}\text { Car- } \\
\text { bon- } \\
\text { ate } \\
\text { (CO }\end{array}$} & \multirow{3}{*}{$\begin{array}{l}\text { Sul- } \\
\text { fate } \\
\left(\mathrm{SO}, \mathrm{s}_{3}\right)\end{array}$} & \multirow{3}{*}{$\begin{array}{c}\text { Chlo- } \\
\text { ride } \\
\text { (CI) }\end{array}$} & \multicolumn{2}{|c|}{$\begin{array}{l}\mathrm{Hardness} \\
{ }_{a s} \mathrm{CaCO}_{3} \\
\end{array}$} & \multirow{3}{*}{$\begin{array}{l}\text { Srecific } \\
\text { conduce- } \\
\text { tance } \\
\text { (mirrs- } \\
\text { mhrs at } \\
25^{\circ} \mathrm{C} \text { ) }\end{array}$} & \multirow{3}{*}{$\mathrm{pH}$} & \multirow{2}{*}{\multicolumn{2}{|c|}{$\begin{array}{c}\text { Waten } \\
\text { temperature }\end{array}$}} \\
\hline & & & & & & & & & & & \multirow{2}{*}{$\begin{array}{l}\mathrm{C}_{\mathrm{a}} \\
\mathrm{M} y\end{array}$} & \multirow{2}{*}{$\begin{array}{c}\text { Non- } \\
\text { car- } \\
\text { bon- } \\
\text { ate }\end{array}$} & & & & \\
\hline & & & & & & & & & & & & & & & on & $o_{r}$ \\
\hline & & & \multicolumn{14}{|c|}{ STREAMS TRIBUTARY TO LAKE MICHIGAN-Continued } \\
\hline 4-1064........ & \multirow{25}{*}{ 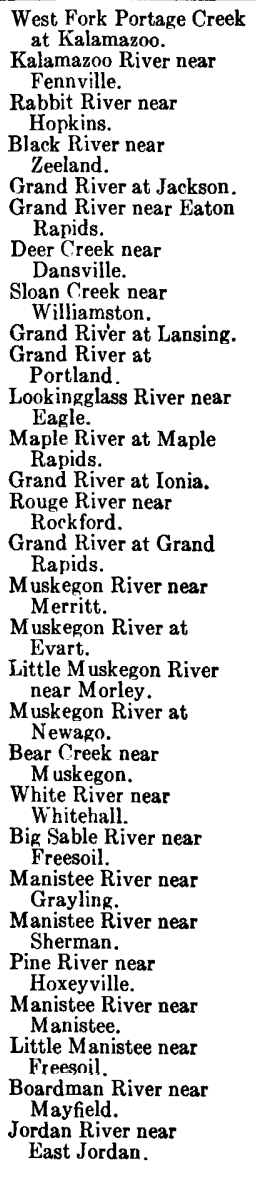 } & NE1/4sec. 5, T. 3 S., R.11 W. & 18.7 & Mar. 14 & 1235 & 13 & 227 & 0 & 22 & 8.0 & 207 & 21 & 400 & 8.2 & 38 & 3.5 \\
\hline $1085 \ldots \ldots$ & & NE1/4sec. 5, T. 2 N., R.14 W. & 1600 & Apr. 12 & 1755 & $\begin{array}{l}3030 \\
795\end{array}$ & ${ }_{254}^{223}$ & 0 & 68 & $\begin{array}{l}16 \\
34\end{array}$ & ${ }_{267}^{252}$ & $\begin{array}{l}69 \\
59\end{array}$ & $\begin{array}{l}495 \\
575\end{array}$ & 8.2 & -. & ........ \\
\hline $1086 \ldots$ & & $\mathrm{SE}^{3} / \mathbf{s e c}^{\mathrm{sec}} 16$, T. 3 N., R.12 W. & 71.4 & $\begin{array}{l}\text { Aug. } 17 \\
\text { Mar. } 28 \\
\text { Aug. } 17\end{array}$ & $\begin{array}{l}1600 \\
1550 \\
1425\end{array}$ & $\begin{array}{l}755 \\
155 \\
14.1\end{array}$ & $\begin{array}{l}254 \\
165 \\
241\end{array}$ & $\begin{array}{l}0 \\
2 \\
4\end{array}$ & $\begin{array}{l}75 \\
40 \\
46\end{array}$ & $\begin{array}{l}34 \\
12 \\
10\end{array}$ & $\begin{array}{l}267 \\
184 \\
242\end{array}$ & $\begin{array}{l}59 \\
46 \\
40\end{array}$ & $\begin{array}{l}575 \\
360 \\
455\end{array}$ & $\begin{array}{l}7.6 \\
8.3 \\
8.5\end{array}$ & $\begin{array}{l}47 \\
74\end{array}$ & $\begin{array}{r}8.5 \\
23.5\end{array}$ \\
\hline 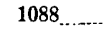 & & NW14sec. 31, T. 5 N., R.14 W. & 65.8 & Apr. $13^{*}$ & 1005 & 30.5 & 184 & 0 & $\begin{array}{l}65 \\
68\end{array}$ & 42 & 242 & 91 & 543 & 8.1 & 69 & 20.5 \\
\hline $\begin{array}{l}1090 \ldots \ldots \\
1110\end{array}$ & & $\begin{array}{l}\mathrm{Sec} .22, \mathrm{~T}, 2 \mathrm{2}, \mathrm{R} .1 \mathrm{~W} \\
\mathrm{NE} 1 / \sec .26, \mathrm{~T}, 2 \mathrm{~N} . \mathrm{R} .3 \mathrm{~W}\end{array}$ & 174 & $\begin{array}{l}\text { Apr. } 6 \\
\text { Apr } 6 \\
\text { A }\end{array}$ & 1540 & 325 & 241 & 3 & 126 & 72 & 347 & 146 & 800 & 8.4 & $\ldots$ & \\
\hline 1115 & & E1/sec. 33, T. 3 N., R.1 E. & 16.3 & & 1800 & 1400 & 201 & 3 & 94 & 19 & 278 & 111 & 530 & 8.4 &.$\cdot$ & .....- \\
\hline 1120 & & E/2 $/ 2 \mathrm{sec} .33$, T. 3 N., R.1 E. & 9.34 & Apr. 0 & 1450 & 23 & 242 & 0 . & 76 & 14 & 297 & 99 & 510 & 8.2 & .... & $\ldots$ \\
\hline $\begin{array}{l}1120 \ldots \\
1130 \ldots\end{array}$ & & $\begin{array}{l}\text { El/28ec. 1, T. } 3 \text { N., R.I W. } \\
\text { NW1/4sec. 9, T. } 4 \text { N., R.2 }\end{array}$ & 1230 & Apr. 5 & $\begin{array}{l}1550 \\
1035\end{array}$ & $\begin{array}{l}10.4 \\
2850\end{array}$ & $\begin{array}{l}239 \\
208\end{array}$ & $\begin{array}{l}3 \\
3\end{array}$ & $\begin{array}{l}58 \\
83\end{array}$ & $\begin{array}{l}18 \\
20\end{array}$ & $\begin{array}{l}286 \\
280\end{array}$ & $\begin{array}{r}88 \\
107\end{array}$ & $\begin{array}{l}520 \\
530\end{array}$ & $\begin{array}{l}8.3 \\
8.4\end{array}$ & $\cdots$ & $\cdots$ \\
\hline & & G $1075 \mathrm{~T} D \mathrm{DT}$ & 1385 & $M=9+3$ & 1600 & 2360 & 211 & $\mathbf{0}$ & 104 & 22 & 298 & 125 & 575 & 8.1 & 54 & 12.0 \\
\hline $1145 \ldots$ & & Sec. 10, T. 5 N., R. 4 W. & 281 & Mar. 31 & 1530 & 673 & 187 & 0 & 87 & 12 & 252 & 99 & 465 & 8.0 & $\ldots$ & ....... \\
\hline $1150 \ldots$. & & Sec. 5, T. 8 N., R.3 W. & 434 & Apr. 15 & 1050 & 629 & 176 & 0 & 45 & 12 & 212 & 68 & 400 & 7.9 & $\ldots$ & $\cdots \cdots$ \\
\hline $\begin{array}{l}1160 \ldots \ldots \\
1185 \ldots \ldots\end{array}$ & & $\begin{array}{l}\text { NW1/4sec. 30, T. } 7 \text { N., R.6 W. } \mathrm{W} . \\
\text { NE1/4sec. 15, , } 8 \text { N., R.11 W. }\end{array}$ & $\begin{array}{r}2840 \\
234\end{array}$ & $\begin{array}{l}\text { Apr. } 15 \\
\text { Apr } 13 \\
\text { Aug. } 18\end{array}$ & $\begin{array}{l}1025 \\
1459 \\
1305\end{array}$ & $\begin{array}{r}4180 \\
1350 \\
169\end{array}$ & $\begin{array}{l}229 \\
190 \\
217\end{array}$ & $\begin{array}{l}4 \\
0 \\
0\end{array}$ & $\begin{array}{l}96 \\
63 \\
59\end{array}$ & $\begin{array}{l}21 \\
15 \\
24\end{array}$ & $\begin{array}{l}\frac{910}{294} \\
234 \\
234\end{array}$ & $\begin{array}{l}98 \\
68 \\
56\end{array}$ & $\begin{array}{l}545 \\
395 \\
500\end{array}$ & $\begin{array}{l}8.4 \\
8.2 \\
7.8\end{array}$ & $\cdots$ & 21.5. \\
\hline 1190 & & 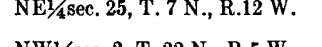 & 4900 & & 1055 & 1100 & 234 & 0 & 54 & 30 & 244 & 52 & 510 & 7.8 & 74 & 23.5 \\
\hline $1210 \ldots$ & & NW1/4sec. 2, T. 22 N., R.5 W. & 309 & Apr. 4 & 1020 & 892 & 60 & 0 & 14 & 4.0 & 58 & 9 & 130 & 7.9 & 36 & 2.0 \\
\hline 1215 & & NE1/4sec. 3 T. 17 N., R.8 W. & 1450 & Mar. 29 & 1155 & 3550 & 94 & 0 & 13 & 7.0 & 87 & 10 & 200 & 8.1 & 35 & 1.5 \\
\hline $\begin{array}{l}1219 \ldots \ldots \\
1220 \ldots \ldots \\
1221 \ldots \ldots\end{array}$ & & $\begin{array}{l}\text { SW1/4se. 24, T. } 13 \text { N., R.9 W. } \\
\text { NE1/4sec. 24, T. } 12 \text { N., R.13 W. } \\
\text { NW1/4sec. 4, T. } 10 \text { N., R.16 W. }\end{array}$ & 2350 & $\begin{array}{l}\text { Mar. } 27 \\
\text { Aug. } 2 \\
\text { Mar. } 20^{*} \\
\text { Aug. } 3 \\
\text { Aug. } 2\end{array}$ & $\begin{array}{l}1600 \\
1600 \\
0810 \\
0900 \\
0895\end{array}$ & $\begin{array}{l}521 \\
59.9 \\
5809 \\
1890 \\
4.58\end{array}$ & $\begin{array}{l}94 \\
202 \\
144 \\
166 \\
110\end{array}$ & $\begin{array}{l}0 \\
0 \\
2 \\
0 \\
0\end{array}$ & $\begin{array}{l}13 \\
12 \\
27 \\
15 \\
17\end{array}$ & $\begin{array}{l}2.0 \\
6.0 \\
18 \\
16 \\
26\end{array}$ & $\begin{array}{l}84 \\
176 \\
152 \\
152 \\
112\end{array}$ & $\begin{array}{l}7 \\
10 \\
30 \\
16 \\
22\end{array}$ & $\begin{array}{l}185 \\
320 \\
333 \\
290 \\
275\end{array}$ & $\begin{array}{l}7.9 \\
7.7 \\
8.3 \\
7.7 \\
7.6\end{array}$ & $\begin{array}{l}34 \\
75 \\
40 \\
70 \\
63\end{array}$ & $\begin{array}{r}1.0 \\
24.0 \\
4.5 \\
21.0 \\
17.0\end{array}$ \\
\hline $1222 \ldots$ & & NW1/4sec. 4, T. 12 N., R.16 W. & 380 & Apr. 18 & & & & & & & & & & & & 17.0 \\
\hline $1230 \ldots$ & & $\mathrm{NE} / 4$ sec. 24, T. 20 N., R.17 W. & 127 & $\begin{array}{l}\text { Aug. 2 } \\
\text { Mar 29* } \\
\text { Aug. } 1\end{array}$ & $\begin{array}{l}1130 \\
1320 \\
1445\end{array}$ & $\begin{array}{r}3360 \\
278 \\
446\end{array}$ & $\begin{array}{r}84 \\
183 \\
84\end{array}$ & $\begin{array}{l}0 \\
0 \\
0\end{array}$ & $\begin{array}{l}10 \\
22 \\
21\end{array}$ & $\begin{array}{l}25.0 \\
25.0\end{array}$ & $\begin{array}{r}74 \\
174 \\
90\end{array}$ & $\begin{array}{l}35 \\
34\end{array}$ & $\begin{array}{l}105 \\
360 \\
192\end{array}$ & $\begin{array}{l}7.9 \\
7.8\end{array}$ & $\begin{array}{l}60 \\
69 \\
38\end{array}$ & $\begin{array}{r}10.5 \\
3.5\end{array}$ \\
\hline $1235 \ldots \ldots$ & & NW1/4sec. 31, T. 27 N., R.4 W. & 159 & $\begin{array}{l}\text { Mar. } 31 \\
\text { Avg } 4\end{array}$ & 1415 & $\frac{113}{281}$ & 168 & 0 & 16 & 170 & $\begin{array}{l}154 \\
128\end{array}$ & $\begin{array}{l}16 \\
0\end{array}$ & 3260 & $\begin{array}{l}8.2 \\
79\end{array}$ & $\begin{array}{l}67 \\
45\end{array}$ & $\begin{array}{c}19.5 \\
7.0\end{array}$ \\
\hline $1240 \ldots$ & & 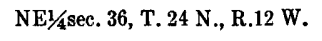 & 900 & Apr. 4 & $\begin{array}{l}1200 \\
1425\end{array}$ & 184 & 180 & 0 & 6.0 & 5.0 & 142 & 0 & 2270 & 8.2 & $\begin{array}{l}60 \\
40\end{array}$ & $\begin{array}{c}15.5 \\
4.5\end{array}$ \\
\hline $1255 \ldots \ldots$ & & $\mathrm{NW} / 4 \mathrm{sec} .20$, T. $21 \mathrm{~N} ., \mathrm{R} .12 \mathrm{~W}$. & 251 & Apr. 6 & - 1950 & 827 & 183 & 0 & 12 & 5.0 & 154 & 4 & 至 & 7.8 & 64 & 18.0 \\
\hline $126 ! \ldots$ & & NW1/4sec. 36, T. 22 N., R.16 W. & 1780 & Apr. $6^{*}$ & 1225 & $\begin{array}{r}230 \\
236 \\
4550\end{array}$ & 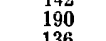 & 0 & 13 & $\begin{array}{l}4.0 \\
4.0\end{array}$ & 154 & 0 & 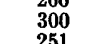 & $\begin{array}{l}8.0 \\
8.0\end{array}$ & 59 & 15.0 \\
\hline $1262 \ldots \ldots$ & & $\mathrm{NE} 1 / 4 \mathrm{sec} .31$, T. 21 N., R.15 W. & 200 & $\begin{array}{l}\text { Mar. } 29 \\
\text { Marg. } 1\end{array}$ & $\begin{array}{l}1200 \\
1600 \\
1225\end{array}$ & $\begin{array}{l}4 \\
1330 \\
425\end{array}$ & $\begin{aligned} \begin{array}{l}100 \\
183 \\
94\end{array} & \end{aligned}$ & $\begin{array}{l}0 \\
0\end{array}$ & 12 & $\begin{array}{r}8.0 \\
8.0 \\
3.0\end{array}$ & $\begin{array}{l}150 \\
156 \\
\$ 99\end{array}$ & 6 & $\begin{array}{l}201 \\
305\end{array}$ & $\begin{array}{l}8.0 \\
8.2\end{array}$ & 71 & $\begin{array}{c}21.5 \\
3.5\end{array}$ \\
\hline $1270 \ldots$ & & NE3/4sec. 21, T. 26 N., R.10 W. & 223 & Apr. $5^{*}$ & 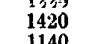 & $\begin{array}{l}463 \\
1169\end{array}$ & 168 & 0 & $\begin{array}{l}11 \\
12 \\
14\end{array}$ & $\begin{array}{l}7.0 \\
2.0\end{array}$ & $\begin{array}{l}143 \\
102\end{array}$ & $\begin{array}{r}12 \\
6\end{array}$ & $\begin{array}{l}730 \\
275 \\
194\end{array}$ & $\begin{array}{l}7.2 \\
8.9\end{array}$ & 64 & 18.0 \\
\hline $1278 \ldots \ldots$ & & NW1/4sec. 7, T. 31 N., R.6 W. & 67.6 & $\begin{array}{l}\text { Mar. } 27 \\
\text { Aug. } 16\end{array}$ & $\begin{array}{l}1120 \\
1730 \\
1110\end{array}$ & $\begin{array}{l}186 \\
416 \\
169\end{array}$ & $\begin{array}{l}171 \\
1166 \\
185\end{array}$ & $\begin{array}{l}2 \\
2 \\
0\end{array}$ & $\begin{array}{r}8.0 \\
14 \\
9.0\end{array}$ & $\begin{array}{l}4.0 \\
2.0 \\
4.0\end{array}$ & $\begin{array}{l}142 \\
130 \\
152\end{array}$ & $\begin{array}{l}0 \\
\mathbf{9} \\
\mathbf{0}\end{array}$ & $\begin{array}{l}265 \\
260 \\
295\end{array}$ & $\begin{array}{l}8.3 \\
8.4 \\
8.0\end{array}$ & $\begin{array}{l}64 \\
38 \\
56 \\
\end{array}$ & $\begin{array}{r}18.0 \\
3.5 \\
13.5 \\
\end{array}$ \\
\hline & & & & S? & IS TRIB & UTARY & O LAKE & JRON & & & & & & & & \\
\hline $4-1280 \ldots$ & $\begin{array}{l}\text { Sturgeon River near } \\
\text { Wolyerine }\end{array}$ & NE1/4sec. 36, T. 34 N., R.3 W. & 170 & & $\begin{array}{l}1055 \\
0955\end{array}$ & 4222 & 176 & $\begin{array}{l}0 \\
0\end{array}$ & 17 & $\begin{array}{l}3.0 \\
4.0\end{array}$ & 152 & $\begin{array}{l}8 \\
6\end{array}$ & 300 & 8.2 & $\begin{array}{l}34 \\
59\end{array}$ & $\begin{aligned} 1.0 \\
15.0\end{aligned}$ \\
\hline & $\begin{array}{l}\text { Pigeon River near } \\
\text { Vanderbilt. }\end{array}$ & SW1/4sec. 9, T. 32 N., R.1 W. & 63.0 & $\begin{array}{l}\text { Apr. } 4 \\
\text { Aug. } 3\end{array}$ & $\begin{array}{l}1330 \\
1245\end{array}$ & $\begin{array}{l}118 \\
63.4\end{array}$ & $\begin{array}{l}170 \\
173\end{array}$ & $\begin{array}{l}0 \\
0\end{array}$ & $\begin{array}{l}13 \\
9.0\end{array}$ & $\begin{array}{l}2.0 \\
4.0\end{array}$ & $\begin{array}{l}152 \\
140\end{array}$ & $\begin{array}{r}13 \\
0\end{array}$ & 270 & $\begin{array}{l}8.1 \\
8.2\end{array}$ & $\begin{array}{l}38 \\
70\end{array}$ & $\begin{array}{r}3.5 \\
21.0\end{array}$ \\
\hline
\end{tabular}




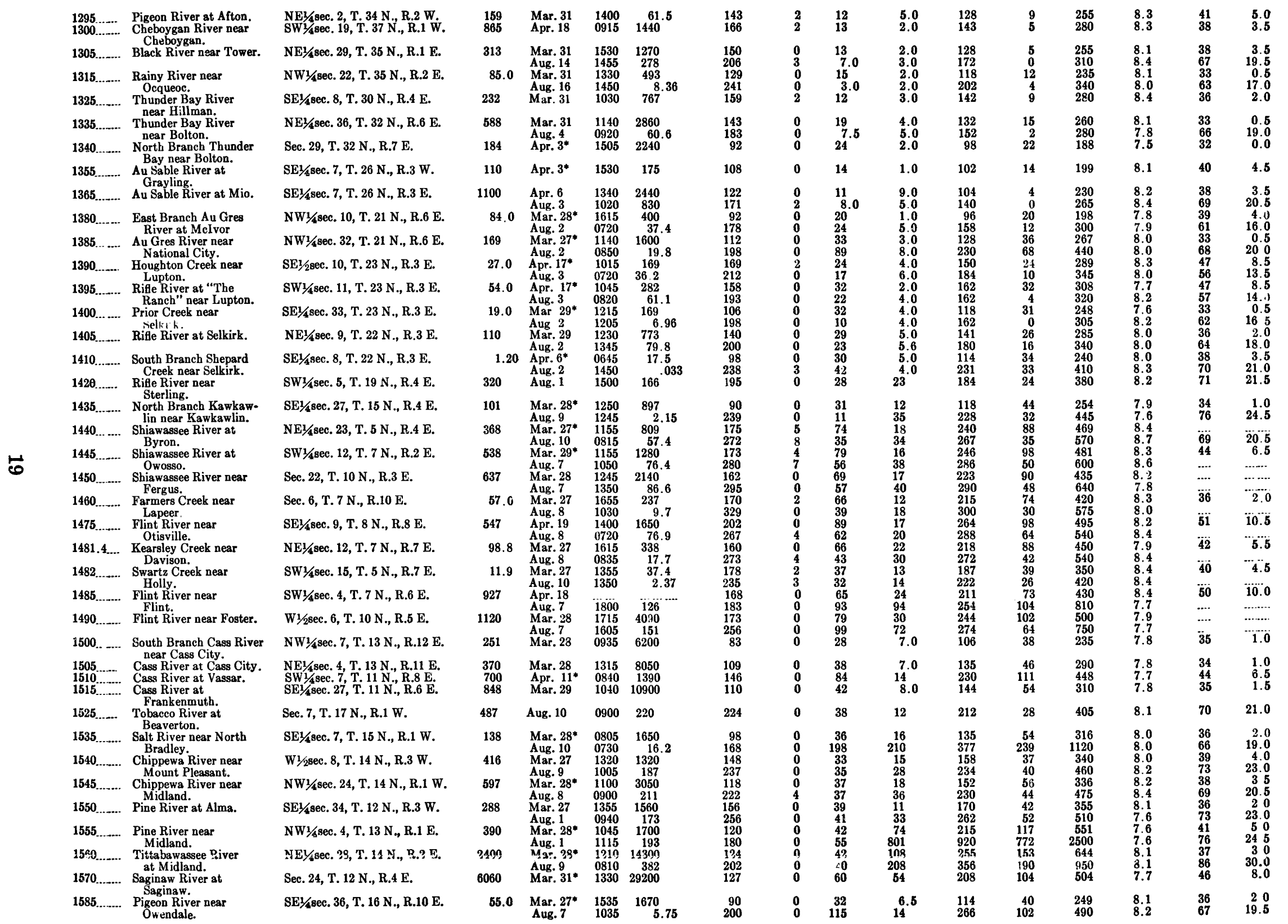


TABLE 1.-Chemical analyses of samples collected from Michigan streams under low-and high-flow conditions, 1967-Continued

\begin{tabular}{|c|c|c|c|c|c|c|c|c|c|c|c|c|c|c|c|c|}
\hline \multirow{3}{*}{$\begin{array}{l}\text { Station } \\
\text { No. }\end{array}$} & \multirow{3}{*}{ Station Name } & \multirow{3}{*}{ Location } & \multirow{3}{*}{$\begin{array}{c}\text { Drain- } \\
\text { age } \\
\text { area } \\
\text { (sq mi) }\end{array}$} & \multirow{3}{*}{$\begin{array}{c}\text { Date } \\
\text { sampled }\end{array}$} & \multirow{3}{*}{ Time } & \multirow{3}{*}{$\begin{array}{l}\text { Dis- } \\
\text { cha.ge } \\
(\mathrm{fs})\end{array}$} & \multirow{3}{*}{$\begin{array}{l}\text { Bicar- } \\
\text { hnnate } \\
\left(\mathrm{HCO}_{3}\right)\end{array}$} & \multirow{3}{*}{$\begin{array}{c}\text { Car- } \\
\text { bnn- } \\
\text { ate } \\
\left(\mathrm{CO}_{3}\right)\end{array}$} & \multirow{3}{*}{$\begin{array}{l}\text { Sul- } \\
\text { fate } \\
\text { (SO .) }\end{array}$} & \multirow{3}{*}{$\begin{array}{l}\text { Chlo- } \\
\text { ride } \\
\text { (Cl) }\end{array}$} & \multicolumn{2}{|c|}{$\begin{array}{l}\text { Hardness } \\
\text { is } \mathrm{CaCO}_{3}\end{array}$} & \multirow{3}{*}{$\begin{array}{c}\text { Specific } \\
\text { conduc. } \\
\text { tance } \\
\text { (micrno- } \\
\text { mhns at } \\
25^{r} \mathrm{C} \text { ) }\end{array}$} & \multirow{3}{*}{$\mathrm{pH}$} & \multirow{2}{*}{\multicolumn{2}{|c|}{$\begin{array}{c}\text { Water } \\
\text { temperature }\end{array}$}} \\
\hline & & & & & & & & & & & \multirow{2}{*}{$\begin{array}{l}\mathrm{Ca} \\
\mathrm{Mg}\end{array}$} & \multirow{2}{*}{$\begin{array}{l}\text { Non- } \\
\text { car-- } \\
\text { bon- } \\
\text { ate }\end{array}$} & & & & \\
\hline & & & & & & & & & & & & & & & ${ }^{\circ} \mathrm{F}$ & ${ }^{\circ} \mathrm{C}$ \\
\hline \multicolumn{17}{|c|}{ STREAMS TRIBUTARY TO ST. CLAIR RIVER } \\
\hline $4-1595 \ldots \ldots$ & Black River near Fargo. & NW1/4sec. 32, T. 8 N., R.16 E. & 480 & Mar. 28 & 1215 & 7030 & 137 & 0 & 26 & $\begin{array}{l}15 \\
64\end{array}$ & 140 & $\begin{array}{r}28 \\
103\end{array}$ & $\begin{array}{l}300 \\
780\end{array}$ & 8.2 & $\cdots$ & ..... \\
\hline $1599 \ldots \ldots$ & Mill Creek near Avoca. & NW1/4sec. 8, T. 7 N., R.15 E. & 169 & & 0930 & 1880 & 85 & 0 & 67 & $\begin{array}{l}04 \\
12\end{array}$ & 152 & 82 & 330 & 8.0 & 38 & 3.5 \\
\hline $1605 \quad 7 \ldots$. & North Branch Belle River & NW1/4sec. 16, T. 7 N., R.12 E. & 18.0 & $\begin{array}{l}\text { Aug. } 9 \\
\text { Mar. } 28\end{array}$ & $\begin{array}{l}0810 \\
1445\end{array}$ & $\begin{array}{l}13.8 \\
920\end{array}$ & $\begin{array}{r}256 \\
62\end{array}$ & $\begin{array}{l}\mathbf{0} \\
0\end{array}$ & $\begin{array}{l}210 \\
136\end{array}$ & $\begin{array}{l}60 \\
25\end{array}$ & $\begin{array}{l}382 \\
228\end{array}$ & $\begin{array}{l}172 \\
177\end{array}$ & $\begin{array}{l}8517 \\
480\end{array}$ & $\begin{array}{l}7.8 \\
8.0\end{array}$ & 41 & 5.0 \\
\hline & at Imlay City. & & 10.0 & $\begin{array}{l}\text { Aug. } 8 \\
\text { A }\end{array}$ & $\begin{array}{l}1430 \\
1235\end{array}$ & 1.42 & 317 & 0 & $\begin{array}{l}150 \\
150\end{array}$ & 300 & 354 & 94 & 1600 & 7.9 & & 5.0 \\
\hline $1606 \ldots \ldots$ & Belle River at Memphis. & SE1/4sec. 35, T. 6 N., R.14 E. & 151 & $\begin{array}{l}\text { Apr. } 20 \\
\text { Aug. } 9\end{array}$ & $\begin{array}{l}1300 \\
1255\end{array}$ & $\begin{array}{l}587 \\
152\end{array}$ & $\begin{array}{l}157 \\
317\end{array}$ & $\begin{array}{l}0 \\
0\end{array}$ & $\begin{array}{l}128 \\
140\end{array}$ & $\begin{array}{l}23 \\
92\end{array}$ & $\begin{array}{l}282 \\
390\end{array}$ & $\begin{array}{l}153 \\
130\end{array}$ & $\begin{array}{l}530 \\
940\end{array}$ & $\begin{array}{l}8.2 \\
7.6\end{array}$ & 50 & 10.0 \\
\hline $1608 \ldots \ldots$ & Sashabaw Creek near & SE1/4sec. 26, T. 4 N., R.9 E. & 21.0 & Apr. 18 & 1120 & 55.9 & 187 & 0 & $\begin{array}{r}140 \\
48\end{array}$ & 13 & 205 & 52 & 385 & 8.2 & $\dddot{49}$ & 9.5 \\
\hline $1609 \ldots$ & $\begin{array}{l}\text { Drayton Plains. } \\
\text { Clinton River near }\end{array}$ & NE1/4sec. 21, T. 3 N., R.9 E. & 79.5 & $\begin{array}{l}\text { Aug. } 16 \\
\text { Apr. } 11\end{array}$ & $\begin{array}{l}1830 \\
1150\end{array}$ & $\underset{116}{2.96}$ & $\begin{array}{l}196 \\
212\end{array}$ & $\begin{array}{l}\mathbf{5} \\
\mathbf{3}\end{array}$ & $\begin{array}{l}42 \\
39\end{array}$ & $\begin{array}{l}18 \\
25\end{array}$ & $\begin{array}{l}220 \\
227\end{array}$ & $\begin{array}{l}54 \\
51\end{array}$ & $\begin{array}{l}420 \\
465\end{array}$ & $\begin{array}{l}8.6 \\
8.4\end{array}$ & $\begin{array}{l}72 \\
45\end{array}$ & $\begin{array}{r}22.0 \\
7.0\end{array}$ \\
\hline & $\begin{array}{l}\text { Drayton Plains. } \\
\text { Clinton }\end{array}$ & $\mathrm{NW} / \mathrm{Mec} 36 \mathrm{~T} 3 \mathrm{~N} \mathrm{~B} 10 \mathrm{~F}$ & & Aug. 31 & 1835 & 15.6 & 220 & 0 & 33 & 40 & 212 & 32 & 495 & 7.8 & 68 & 20.0 \\
\hline $1610 \ldots \ldots$ & $\begin{array}{l}\text { Clinton River at } \\
\text { Auburn Heights. }\end{array}$ & NW1/48ec. 36, T. 3 N., R.10 E. & 123 & $\begin{array}{l}\text { Apr. } 19 \\
\text { Aug. } 16\end{array}$ & $\begin{array}{l}1005 \\
1745\end{array}$ & 209 & $\begin{array}{l}210 \\
168\end{array}$ & $\begin{array}{l}0 \\
2\end{array}$ & $\begin{array}{l}66 \\
93\end{array}$ & $\begin{array}{r}74 \\
114\end{array}$ & $\begin{array}{l}256 \\
282\end{array}$ & $\begin{array}{r}84 \\
142\end{array}$ & $\begin{array}{l}680 \\
850\end{array}$ & $\begin{array}{l}82 \\
83\end{array}$ & 79 & 260 \\
\hline $1611 \ldots \ldots$ & Galloway Creek near & SE1/4sec. 18, T. 3 N., R.11 E. & 17.8 & Apr. 11 & 0900 & 20.6 & 256 & 4 & 97 & 104 & 370 & 158 & 865 & 8.3 & $\ldots$ & co.v \\
\hline $1615 \ldots \ldots$ & $\begin{array}{l}\text { Auburn Heights. } \\
\text { Paint Creek near }\end{array}$ & NE1/4sec. 13, T. 4 N., R.10 E. & 38.9 & $\begin{array}{l}\text { Aug. } 31 \\
\text { Apr. } 11\end{array}$ & $\begin{array}{l}1635 \\
1350\end{array}$ & $68 \cdot 56$ & $\begin{array}{l}334 \\
213\end{array}$ & $\begin{array}{l}0 \\
4\end{array}$ & $\begin{array}{r}125 \\
62\end{array}$ & $\begin{array}{l}64 \\
18\end{array}$ & $\begin{array}{l}326 \\
246\end{array}$ & $\begin{array}{l}52 \\
68\end{array}$ & $\begin{array}{l}820 \\
480\end{array}$ & $\begin{array}{l}7.6 \\
8.5\end{array}$ & $\dddot{48}$ & 9.0 \\
\hline & Lake Orion. & & & Aug. 31 & 1455 & 11.7 & 222 & 0 & 45 & 24 & 228 & 46 & 478 & 7.7 & .... & ........ \\
\hline $1615,4 \ldots$ & $\begin{array}{l}\text { Paint Creek at } \\
\text { Rochester. }\end{array}$ & SE1/4sec. 10, T. 3 N., R.11 E. & 71.3 & $\begin{array}{l}\text { Apr. } 19 \\
\text { Aug. } 16\end{array}$ & $\begin{array}{l}0920 \\
1605\end{array}$ & 223 & $\begin{array}{l}220 \\
260\end{array}$ & $\begin{array}{l}0 \\
5\end{array}$ & $\begin{array}{l}\mathbf{5 8} \\
\mathbf{3 8}\end{array}$ & 18 & $\begin{array}{l}240 \\
258\end{array}$ & $\begin{array}{l}60 \\
42\end{array}$ & $\begin{array}{l}470 \\
530\end{array}$ & $\begin{array}{l}8.0 \\
85\end{array}$ & 70 & 21.0 \\
\hline $1615.8 \ldots$ & Stony Creek near & SW1/4sec. 31, T. 5 N., R.12 E. & 25.6 & Apr. 11 & 1620 & 455 & 205 & 4 & 40 & 13 & 215 & 44 & 400 & 85 & .... & $\ldots .0$ \\
\hline $1616 \ldots \ldots$ & $\begin{array}{l}\text { Romeoe. } \\
\text { West Branch Stony }\end{array}$ & SE1/4sec. 5, T. 4 N., R.11 E. & 14.8 & $\begin{array}{l}\text { Aug. } 31 \\
\text { Apr. } 11\end{array}$ & $\begin{array}{l}1155 \\
1545\end{array}$ & $\begin{array}{r}3.52 \\
12.1\end{array}$ & $\begin{array}{l}334 \\
196\end{array}$ & $\begin{array}{l}\mathbf{0} \\
\mathbf{2}\end{array}$ & $\begin{array}{l}39 \\
27\end{array}$ & $\begin{array}{r}16 \\
8.0\end{array}$ & $\begin{array}{l}306 \\
188\end{array}$ & $\begin{array}{l}32 \\
25\end{array}$ & $\begin{array}{l}580 \\
350\end{array}$ & $\begin{array}{l}8.0 \\
83\end{array}$ & $\cdots$ & $\cdots \cdots$ \\
\hline $1618 \ldots \ldots$ & $\begin{array}{l}\text { Creek near L i keville. } \\
\text { Stony Creek near }\end{array}$ & SW1/4sec. 31, T. 4 N., R.12 E. & 68.0 & Apr. 10 & 1640 & 137 & 200 & 2 & 40 & 14 & 215 & 49 & 400 & 8.3 & $\ldots$. & $\ldots \ldots$ \\
\hline $1620 \ldots \ldots$ & $\begin{array}{l}\text { Washington. } \\
\text { Red Run near Royal }\end{array}$ & NE1/4sec. 12, T. 1 N., R.11 E. & 36.5 & $\begin{array}{l}\text { Aug. } 31 \\
\text { Apr. } 13\end{array}$ & $\begin{array}{l}1000 \\
1600\end{array}$ & $\begin{array}{r}588 \\
.48\end{array}$ & $\begin{array}{l}244 \\
470\end{array}$ & $\begin{array}{l}\mathbf{0} \\
\mathbf{0}\end{array}$ & $\begin{array}{r}35 \\
290\end{array}$ & $\begin{array}{r}18 \\
406\end{array}$ & $\begin{array}{l}235 \\
790\end{array}$ & $\begin{array}{r}35 \\
405\end{array}$ & $\begin{array}{r}455 \\
2500\end{array}$ & $\begin{array}{l}7.9 \\
8.0\end{array}$ & $\cdots$. & $\ldots \ldots .$. \\
\hline $1629 \ldots \ldots$ & $\begin{array}{l}\text { Oak. } \\
\text { Big Beaver Creek near }\end{array}$ & SW1/4sec. 33, T. 2 N., R.12 E. & 23.5 & Apr. 13 & 1420 & 12.7 & 204 & $\mathbf{5}$ & 94 & 112 & 308 & 136 & 900 & 84 & & \\
\hline $1029 \ldots . . .$. & Warren. & 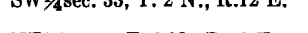 & & Aug. 24 & 1555 & 12.87 & 270 & $\mathbf{5}$ & $\begin{array}{l}94 \\
58\end{array}$ & 190 & 333 & 107 & 1220 & 84 & 69 & 20.5 \\
\hline $1634 \ldots \ldots$ & Plum Brook at Utica. & NE1/4sec. 7, T. 2 N., R.12 E. & 16.1 & Apr. 13 & 1325 & 107 & 192 & 2 & 94 & 120 & 307 & 147 & 900 & 83 & 97 & 195 \\
\hline $1640 \ldots \ldots$ & Clinton River near & NW1/4sec. 20, T. 2 N., R.13 E. & 445 & $\begin{array}{l}\text { Aug. } 25 \\
\text { Apr } 20\end{array}$ & $\begin{array}{l}1150 \\
1135\end{array}$ & $811^{27}$ & $\begin{array}{l}256 \\
219\end{array}$ & $\mathbf{3}_{3}^{0}$ & $\begin{array}{l}88 \\
60\end{array}$ & $\begin{array}{r}156 \\
51\end{array}$ & $\begin{array}{l}366 \\
269\end{array}$ & $\begin{array}{r}156 \\
81\end{array}$ & $\begin{array}{r}1090 \\
610\end{array}$ & $\begin{array}{l}7.8 \\
8.3\end{array}$ & 67 & $\begin{array}{l}19.5 \\
\ldots \ldots \ldots\end{array}$ \\
\hline & Fraser. & & & Aug. 16 & 1058 & 152 & 215 & 0 & 67 & 64 & 244 & 68 & 690 & 79 & . & ….... \\
\hline $1640.1 \ldots$ & North Branch Clinton & NE1/4sec. 28, T. 6 N., R.12 E. & 9.56 & Apr. 21 & 0750 & 165 & 231 & 3 & 64 & 16 & 267 & 75 & 530 & 8.3 & 47 & 8.5 \\
\hline $1640.5 \ldots$ & $\begin{array}{l}\text { River at Almont. } \\
\text { North Branch Clinton }\end{array}$ & NW1/4ec. 31, T. 5 N., R.13 E. & 49.7 & $\begin{array}{l}\text { Aug. } 8 \\
\text { Apr. } 21\end{array}$ & $\begin{array}{l}1410 \\
1225\end{array}$ & ${ }_{92}^{170}$ & $\begin{array}{l}220 \\
247\end{array}$ & $\begin{array}{l}\mathbf{0} \\
\mathbf{3}\end{array}$ & $\begin{array}{l}48 \\
92\end{array}$ & $\begin{array}{l}18 \\
26\end{array}$ & $\begin{array}{l}214 \\
317\end{array}$ & $\begin{array}{r}34 \\
112\end{array}$ & $\begin{array}{l}620 \\
640\end{array}$ & $\begin{array}{l}8.0 \\
8.3\end{array}$ & $\dddot{5} 1$ & 10.5 \\
\hline & near Romeo. & & 20.8 & Aug. 8 & 1530 & 9.45 & 306 & 4 & 78 & 34 & 330 & 76 & 665 & 8.3 & $\dddot{z} 1$ & \\
\hline $1641 \ldots \ldots$ & East Pond Creek at & SE1/4sec. 27, T. 5 N., R.12 E. & 21.8 & Apr. 21 & 1150 & 718 & 204 & 2 & 42 & 22 & 216 & 47 & 430 & 8.3 & 51 & 10.5 \\
\hline $1642 \ldots \ldots$ & $\begin{array}{l}\text { Romeo. } \\
\text { Coon Creek near }\end{array}$ & SW1/4sec. 1, T. 4 N., R.13 E. & 10.0 & $\begin{array}{l}\text { Aug. } 8 \\
\text { A r. } 12\end{array}$ & $\begin{array}{l}1450 \\
1105\end{array}$ & $\begin{array}{l}5.46 \\
7.40\end{array}$ & $\begin{array}{l}295 \\
186\end{array}$ & $\begin{array}{l}\mathbf{4} \\
\mathbf{2}\end{array}$ & $\begin{array}{l}23 \\
86\end{array}$ & $\begin{array}{l}32 \\
28\end{array}$ & $\begin{array}{r}250 \\
270\end{array}$ & 115 & $\begin{array}{l}540 \\
610\end{array}$ & $\begin{array}{l}8.3 \\
8.3\end{array}$ & $\cdots$ & $\ldots \ldots$. \\
\hline & Armada. & & & Aug. 30 & 1625 & 1.01 & 310 & 0 & 84 & 26 & 332 & 78 & 640 & 8.0 & ..... & .......... \\
\hline 1642.5 & Tupper Brook at Ray & NW1/4sec. 23, T. 4 N., R.13 E. & 8.62 & Apr. 12 & $\ldots \ldots$ & 10.6 & 205 & 0 & 89 & 28 & 282 & 114 & 600 & 8.2 & .... & $\cdots \cdots$ \\
\hline $1643 \ldots \ldots$ & $\begin{array}{l}\text { Center. } \\
\text { East Branch Coon }\end{array}$ & NE1/4sec. 23, T. 5 N., R.13 E. & 13.0 & $\begin{array}{l}\text { Aug. } 30 \\
\text { Apr. } 21\end{array}$ & $\ddot{0} 9 \dddot{50}$ & $10.3^{05}$ & $\begin{array}{r}1151 \\
251\end{array}$ & $\begin{array}{l}\mathbf{0} \\
\mathbf{0}\end{array}$ & $\begin{array}{l}115 \\
120\end{array}$ & $\begin{array}{r}294 \\
33\end{array}$ & $\begin{array}{l}518 \\
358\end{array}$ & $\begin{array}{r}0 \\
152\end{array}$ & $\begin{array}{r}2400 \\
670\end{array}$ & $\begin{array}{l}7.1 \\
8.2\end{array}$ & 50 & 10.0 \\
\hline & Creek at Armada. & & & Aug. 8 & 1623 & .36 & 354 & 0 & 113 & 34 & 384 & 94 & 755 & 8.1 & $\ldots$ & $\ldots . .$. \\
\hline $1643.5 \ldots$ & Highbank Creek near & NW1/4sec. 6, T. 4 N., R.14 E. & 14.9 & Apr. 12 & 0950 & 4.36 & 193 & 0 & 93 & 56 & 288 & 130 & 650 & 8.2 & $\dddot{s i}$ & 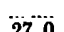 \\
\hline $1645 \ldots \ldots$ & $\begin{array}{l}\text { Armada. } \\
\text { North Branch Clinton }\end{array}$ & NW1/4sec. 2, T. 2 N., R.13 E. & 199 & $\begin{array}{l}\text { Aug. } 30 \\
\text { Apr. } 12\end{array}$ & $\begin{array}{l}1720 \\
1625\end{array}$ & $180^{.37}$ & $\begin{array}{l}224 \\
184\end{array}$ & $\begin{array}{l}0 \\
0\end{array}$ & $\begin{array}{r}148 \\
91\end{array}$ & $\begin{array}{l}62 \\
38\end{array}$ & $\begin{array}{l}340 \\
272\end{array}$ & $\begin{array}{l}156 \\
121\end{array}$ & $\begin{array}{l}\mathbf{7 5 0} \\
\mathbf{5 7 0}\end{array}$ & $\begin{array}{l}7.8 \\
8.2\end{array}$ & $\ldots$ & .......... \\
\hline & River near Mount & & & Aug. 29 & 0110 & 26.2 & 261 & 0 & 76 & 48 & 284 & 70 & 620 & 7.6 & ... & …..... \\
\hline $1646 \ldots$ & Middle Branch Clinton & SE1/4sec. 2, T. 3 N., R.12 E. & 22.2 & Apr. 20 & 0920 & 26 & 213 & $\mathbf{0}$ & 66 & 44 & 274 & 99 & 640 & 8.2 & 44 & 6.5 \\
\hline & River near Macomb. & & & Aug. 16 & 1420 & 2.91 & 266 & 0 & 55 & 42 & 268 & 50 & 630 & 7.9 & $\ldots$ & ........ \\
\hline $1648 \ldots \ldots$ & Middle Branch Clinton & SW1/4sec. 5, T. 3 N., R.13 E. & 41.0 & Apr. 12 & 1500 & 269 & 165 & 2 & 80 & 46 & 258 & 120 & 700 & $\begin{array}{r}83 \\
70\end{array}$ & $\ldots$. & ......... \\
\hline $1655 \ldots \ldots$ & $\begin{array}{l}\text { River at Macomb. } \\
\text { Clinton River at Mount }\end{array}$ & T. 2 N., R.13 E. & 734 & $\begin{array}{l}\text { Aug. } 30 \\
\text { Aug. } 29\end{array}$ & $\begin{array}{l}1345 \\
1240\end{array}$ & & $\begin{array}{l}217 \\
246\end{array}$ & $\begin{array}{l}0 \\
0\end{array}$ & $\begin{array}{l}55 \\
57\end{array}$ & $\begin{array}{l}42 \\
58\end{array}$ & $\begin{array}{l}248 \\
248\end{array}$ & $\begin{array}{l}70 \\
46\end{array}$ & $\begin{array}{l}560 \\
645\end{array}$ & $\begin{array}{l}7.8 \\
7.6\end{array}$ & .... & …..... \\
\hline & $\begin{array}{l}\text { Clemons at Moravian } \\
\text { Drive. }\end{array}$ & & & & & & & & & & & & & & & \\
\hline
\end{tabular}


STREAMS TRIBUTARY TO DETROIT RIVER

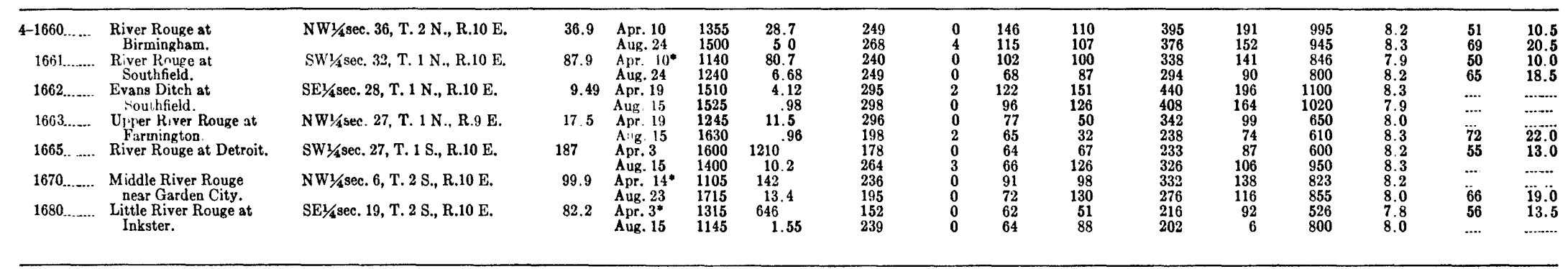

STREAMS TRIBUTARY TO LAKE ERIE

4-1695...... Huron River at SE1/4sec. 10, T. 2 N., R.8 E.

1700 Commerce. Her.

1705....... Huron River near New

1715...... Ore Creek near

1720...... Huron River at

1725....... Portage River near

1730....... Huron River near

1735....... Mill Creek near

1745....... Huron River at Ann

1757....... River Raisin near

$1760 \ldots . . . . \quad \begin{gathered}\text { Tecumseh. } \\ \text { River Raisin near }\end{gathered}$

Adrian.
1765........ River Raisin near
Monroe, on Ida May Monroe, on
bee Road.

$\begin{array}{clcc}49.6 & \text { Apr. 6 } & 1515 & 86.3 \\ 125 & \text { Aug. 16 } & 0850 & 13.7 \\ & \text { Apr. } 6 & 1420 & 173 \\ 143 & \text { Aug. 17 } & 1240 & 39.4 \\ & \text { Apr. } 6 & 1220 & 46.8 \\ 31.0 & \text { Aug. 17 } & 1445 & 40.3 \\ & \text { Mar. 31 } & 1630 & 34.7 \\ 299 & \text { Aug. 17 } & 1710 & 4.38 \\ & \text { Kar. } 31 & 1555 & 382 \\ 79.0 & \text { Mar. } 31 & 1115 & 120 \\ & \text { Aug. 18 } & 1010 & 2.13 \\ 506 & \text { Mar. 31 } & 1235 & 854 \\ & \text { Aug. 18 } & 1300 & 81.8 \\ 134 & \text { Apr. 26 } & 1000 & 110 \\ 711 & \text { Aug. 22 } & 1530 & 17.4 \\ 711 & \text { Apr. 21 } & 0925 & 668 \\ 266 & \text { Aug. 22 } & 1700 & 81.4 \\ & \text { Apr. 17 } & 1530 & 404 \\ 455 & \text { Aup. 14 } & 1210 & 14.2 \\ & \text { Apr. 17 } & 1245 & 597 \\ 1034 & \text { Aug. 14 } & 1200 & 47.9 \\ & \text { Apr. } 4 * & 1605 & 3110 \\ & \text { Aug. 15 } & 0930 & 66.9 \\ & & & \end{array}$

216
225
229
232
223
198
218
249
214
201
185
202
210
307
243
230
220
253
212
245
273
178
168

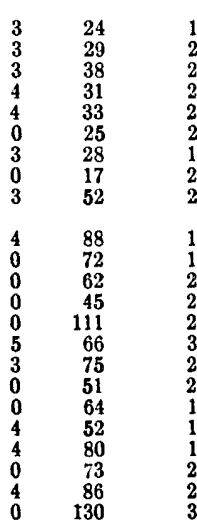

\begin{tabular}{|c|c|c|c|c|c|c|}
\hline $\begin{array}{l}17 \\
25 \\
26 \\
29 \\
26 \\
26 \\
15 \\
20 \\
27\end{array}$ & $\begin{array}{l}207 \\
224 \\
232 \\
228 \\
222 \\
190 \\
220 \\
222 \\
240\end{array}$ & $\begin{array}{l}27 \\
36 \\
41 \\
32 \\
36 \\
28 \\
39 \\
18 \\
62\end{array}$ & $\begin{array}{l}410 \\
560 \\
470 \\
480 \\
465 \\
410 \\
400 \\
450 \\
500\end{array}$ & $\begin{array}{l}8.4 \\
8.3 \\
8.3 \\
8.5 \\
8.4 \\
7.7 \\
8.4 \\
7.7 \\
8.4\end{array}$ & $\begin{array}{l}79 \\
\ldots \ldots \\
\ldots \\
\ldots\end{array}$ & $\begin{array}{c}\dddot{26.0} \\
\ldots \ldots \\
\cdots \ldots\end{array}$ \\
\hline $\begin{array}{l}14 \\
15 \\
24 \\
26 \\
27 \\
34 \\
22 \\
28 \\
12 \\
17 \\
16 \\
28 \\
28 \\
38\end{array}$ & $\begin{array}{l}274 \\
228 \\
242 \\
220 \\
384 \\
270 \\
276 \\
236 \\
274 \\
238 \\
294 \\
302 \\
280 \\
268\end{array}$ & $\begin{array}{r}106 \\
76 \\
76 \\
48 \\
132 \\
66 \\
85 \\
56 \\
67 \\
56 \\
91 \\
78 \\
127 \\
130\end{array}$ & $\begin{array}{l}520 \\
485 \\
488 \\
465 \\
755 \\
700 \\
520 \\
520 \\
500 \\
545 \\
545 \\
640 \\
561 \\
690\end{array}$ & $\begin{array}{l}8.4 \\
8.2 \\
8.2 \\
8.2 \\
8.2 \\
8.5 \\
8.3 \\
8.2 \\
8.2 \\
8.5 \\
8.4 \\
8.0 \\
8.4 \\
7.6\end{array}$ & $\begin{array}{l}78 \\
68 \\
52 \\
72 \\
61\end{array}$ & $\begin{array}{r}9.0 \\
20.0 \\
11.0 \\
22.0 \\
16.0 \\
15.5 \\
\ldots . . \\
\ldots . .\end{array}$ \\
\hline
\end{tabular}

- Laboratory analysis. 


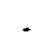

